\title{
Ebola virus antibody decay-stimulation in a high proportion of survivors
}

https://doi.org/10.1038/s41586-020-03146-y

Received: 19 November 2019

Accepted: 18 December 2020

Published online: 27 January 2021

Check for updates

\author{
Charlene Adaken ${ }^{1,2,32}$, Janet T. Scott ${ }^{2,3,4,32}$, Raman Sharma ${ }^{5,32}$, Robin Gopal ${ }^{6}$, Steven Dicks ${ }^{7,8}$, \\ Saidia Niazi ${ }^{7,8}$, Samreen Ijaz ${ }^{7,8}$, Tansy Edwards ${ }^{9}$, Catherine C. Smith ${ }^{3,10,29}$, Christine P. Cole ${ }^{11,30}$, \\ Philip Kamara ${ }^{12}$, Osman Kargbo ${ }^{12}$, Heidi A. Doughty ${ }^{13,14}$, Johan van Griensven ${ }^{15}$, Peter W. Horby ${ }^{16}$, \\ Sahr M. Gevao ${ }^{12,17}$, Foday Sahr ${ }^{17,18}$, the Ebola-CP Consortium* ${ }^{*}$, Richard J. Dimelow ${ }^{19}$, \\ Richard S. Tedder ${ }^{2,7,8,31,33}$, Malcolm G. Semple ${ }^{2,3,33}$, William A. Paxton ${ }^{1,2,33}$ \& Georgios Pollakis ${ }^{1,2,33 凶}$
}

Neutralizing antibody function provides a foundation for the efficacy of vaccines and therapies ${ }^{1-3}$. Here, using a robust in vitro Ebola virus (EBOV) pseudo-particle infection assay and a well-defined set of solid-phase assays, we describe a wide spectrum of antibody responses in a cohort of healthy survivors of the Sierra Leone EBOV outbreak of 2013-2016. Pseudo-particle virus-neutralizing antibodies correlated with total anti-EBOV reactivity and neutralizing antibodies against live EBOV. Variant EBOV glycoproteins (1995 and 2014 strains) were similarly neutralized. During longitudinal follow-up, antibody responses fluctuated in a 'decay-stimulation-decay' pattern that suggests de novo restimulation by EBOV antigens after recovery. A pharmacodynamic model of antibody reactivity identified a decay half-life of 77-100 days and a doubling time of $46-86$ days in a high proportion of survivors. The highest antibody reactivity was observed around 200 days after an individual had recovered. The model suggests that EBOV antibody reactivity declines over 0.5-2 years after recovery. In a high proportion of healthy survivors, antibody responses undergo rapid restimulation. Vigilant follow-up of survivors and possible elective de novo antigenic stimulation by vaccine immunization should be considered in order to prevent EBOV viral recrudescence in recovering individuals and thereby to mitigate the potential risk of reseeding an outbreak.
Limited EBOV outbreaks have been recorded since $1976^{1}$. The much larger 2013-2016 West African epidemic (28,610 cases) and the ongoing 2018 Eastern Zaire outbreak (3,188 cases as of September 2019) (https://www.who.int/emergencies/diseases/Ebola/drc-2019) in the Democratic Republic of the Congo (DRC) have been more extensive. These larger outbreaks have indicated that the virus can persist in some individuals, with the potential for subsequent viral transmis$\operatorname{sion}^{2}$. Because the number of Ebola outbreaks has been small, we have limited understanding of natural induced immune responses, and our knowledge of vaccine-induced responses comes largely from animal models ${ }^{3}$. These models have indicated that total levels of IgG-binding antibodies can correlate with protection and with neutralizing antibody ( $\mathrm{nAb}$ ) responses, which can typically be low.
Outbreaks in humans have provided valuable information regarding therapeutic ${ }^{4}$ and vaccine intervention strategies ${ }^{5-7}$ for EBOV. More recently, nAbs have been the focus of therapeutic development ${ }^{8-12}$. A cocktail of monoclonal antibodies (mAbs) was administered during the 2013-2016 outbreak ${ }^{12,13}$, and trials conducted in the DRC showed evidence of efficacy ${ }^{14}$. In early 2015, two related studies (Ebola-Tx ${ }^{15}$ and Ebola- $\mathrm{CP}^{16}$ ) were established to recruit apparently health survivors of EBOV with the intent of using their convalescent plasma (CP) to treat disease ${ }^{4,16,17}$. We used CP from the donors of the Ebola-CP study (Supplementary Table 1a), in which samples were collected longitudinally (30-500 days) to better ascertain how nAb responses evolve. Such responses have previously been studied in both humans and primates with broad nAb activity ${ }^{4,18-20}$.

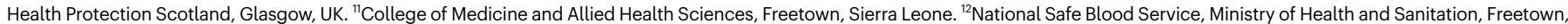
Sierra Leone. ${ }^{13}$ National Health Service Blood and Transplant, Birmingham, UK. ${ }^{14}$ College of Medical and Dental Sciences, University of Birmingham, Birmingham, UK. ${ }^{15}$ Institute of Tropical Medicine, Antwerp, Belgium. ${ }^{16}$ Centre for Tropical Medicine and Global Health, University of Oxford, Oxford, UK. ${ }^{17}$ College of Medicine and Allied Health Sciences, University of Sierra Leone Freetown, Sierra Leone. ${ }^{18} 34$ Military Hospital, Republic of Sierra Leone Armed Forces, Freetown, Sierra Leone. ${ }^{19}$ GlaxoSmithKline, Stevenage, UK. ${ }^{29}$ Present address: Oxford Vaccine Group, University of Oxford, Centre for Clinical Vaccinology and Tropical Medicine, Oxford, UK. ${ }^{30}$ Present address: Royal Liverpool and Broadgreen University Hospitals NHS Trust, Liverpool, UK. ${ }^{31}$ Present address: Division of Infectious Diseases, Imperial College London, London, UK. ${ }^{32}$ These authors contributed equally: Charlene Adaken, Janet T. Scott, Raman Sharma. ${ }^{33}$ These authors jointly supervised this work: Richard S. Tedder, Malcolm G. Semple, William A. Paxton, Georgios Pollakis. *A list of authors and their affiliations appears at the end of the paper.

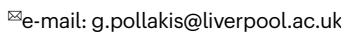


We initially developed a range of solid-phase enzyme-linked immunoassays (EIAs), based on the Mayinga EBOV strain recombinant antigen, to characterize antibody responses in potential donors of therapeutic $\mathrm{CP}^{21}$. To circumvent the difficulty of using replication-competent EBOV in expanding the analysis to characterize neutralization responses, we used single-round infectious pseudo-particle viruses (PVVs; see Methods). Optimal virus production and infectivity were identified by limiting dilution of a plasmid expressing variant EBOV glycoprotein from the 2014 strain (EBOV14-GP; Extended Data Fig. 1a). Glycoproteins from three EBOV strains were used for PPV production; the early 2014 epidemic strain (pEBOV14-GP; accession KP096421 in NCBI database Nucleotide (https://www.ncbi.nlm.nih.gov/nucleotide/) ${ }^{22}$; a modified variant (pEBOV14m-GP) with mutations that appeared early during the outbreak (Fig. 1b, Supplementary Table 2); and the 1995 Kikwit strain (pEBOV95-GP; accession KC242799) ${ }^{23}$, which was represented in the vaccine administered latterly in the 2013-2016 outbreak. EBOV14-GP PPVs demonstrated consistently lower infectivity than the other strains (Fig. 1a), presumably because of the T544I amino acid mutation previously described ${ }^{24}$. The A82V alteration (pEBOV14m-GP), which appeared early in the epidemic and was subsequently found in more than $90 \%$ of $2013-2016$ isolates, was also reported to have a higher infectivity profile ${ }^{25}$. Notably, this genotype was not associated with altered disease pathogenicity in a primate model system ${ }^{26}$.

We used the above PPV infection assay to quantify nAb responses in $\mathrm{CP}$ donors (using limiting dilutions of plasma). To identify non-specific neutralization effects, we tested EBOV antibody-negative plasmas $(n=6)$ to find the range of non-specific inhibition (Extended Data Fig. 1b), and CPs with results that fell within this range were considered to lack neutralizing potential. We also used PPVs expressing the HIV-1 envelope protein to test a high-titre EBOV antibody-positive plasma that was within the non-neutralizing range (Extended Data Fig. 1b). The WHO Anti-EBOV Convalescent Plasma International Reference Panel (NIBSC 16/344) was used to demonstrate the neutralizing potential of EBOV antibody-positive sera (half-maximal inhibitory concentration $\left(\mathrm{IC}_{50}\right)$ range, 6.33-7.01 $\log _{2}$ [plasma dilution]; Extended Data Fig. 1c), which was comparable to previously published values ${ }^{27}$. We tested the robustness of the assay using plasma from EBOV survivors to inhibit the three PPV strains produced, each in different batches with the assay repeated in two biologically independent experiments (Extended Data Fig. 1d).

CPs $(n=52)$ demonstrated a wide range of neutralization potential (Supplementary Table $1 \mathrm{~b}-\mathrm{d}$ ); however, they had comparable profiles when assayed using all three EBOV PPV strains (Extended Data Fig. $2 \mathrm{a}-\mathrm{c})$. Half-maximal $\left(\mathrm{IC}_{50}\right)$ and $70 \%\left(\mathrm{IC}_{70}\right)$ inhibitory concentrations were correlated (Extended Data Fig. 3). Within this cohort we found no differences in neutralizing titres among the three virus strains (Fig. 1c, Extended Data Fig. 1e). Neutralization of PPVs expressing pEBOV14-GP, with the lower infectivity profile (Fig. 1a), did not differ from that of the pEBOV95-GP strain isolated twenty years earlier, or the pEBOV14m-GP strain carrying early epidemic mutations, including the $A 82 \mathrm{~V}$ variant associated with higher infectivity. However, individual CPs that had high $\mathrm{IC}_{50}$ and $\mathrm{IC}_{70}$ values against one virus strain did not necessarily neutralize the other two (Fig. 1d, Extended Data Fig. 1f), potentially highlighting epitope diversity among individual participants as well as virus strains. A subset of donor CPs $(n=5)$ with sequential samplings (totalling $n=30$; Supplementary Table 1e) were assayed against the replication-competent EBOV (RCE) Makona 2014 isolate. There was a significant correlation between the two neutralization platforms (Fig. 1e, Extended Data Fig. 1g; $r=0.52, P<0.0001$ ). In addition, our neutralization data demonstrated a similarly significant correlation with total anti-EBOV reactivity measured using the double antigen bridging assay (DABA) (Fig. If $\left(r=0.50, P<0.0001, \mathrm{IC}_{50}\right)$, Extended Data Fig. $\left.1 \mathrm{~h}\left(r=0.55, P<0.0001, \mathrm{IC}_{70}\right)\right)$. These data corroborate previous results ${ }^{21}$ and further validate the PPV neutralization platform used here. The RCE and PPV assays demonstrated a stronger

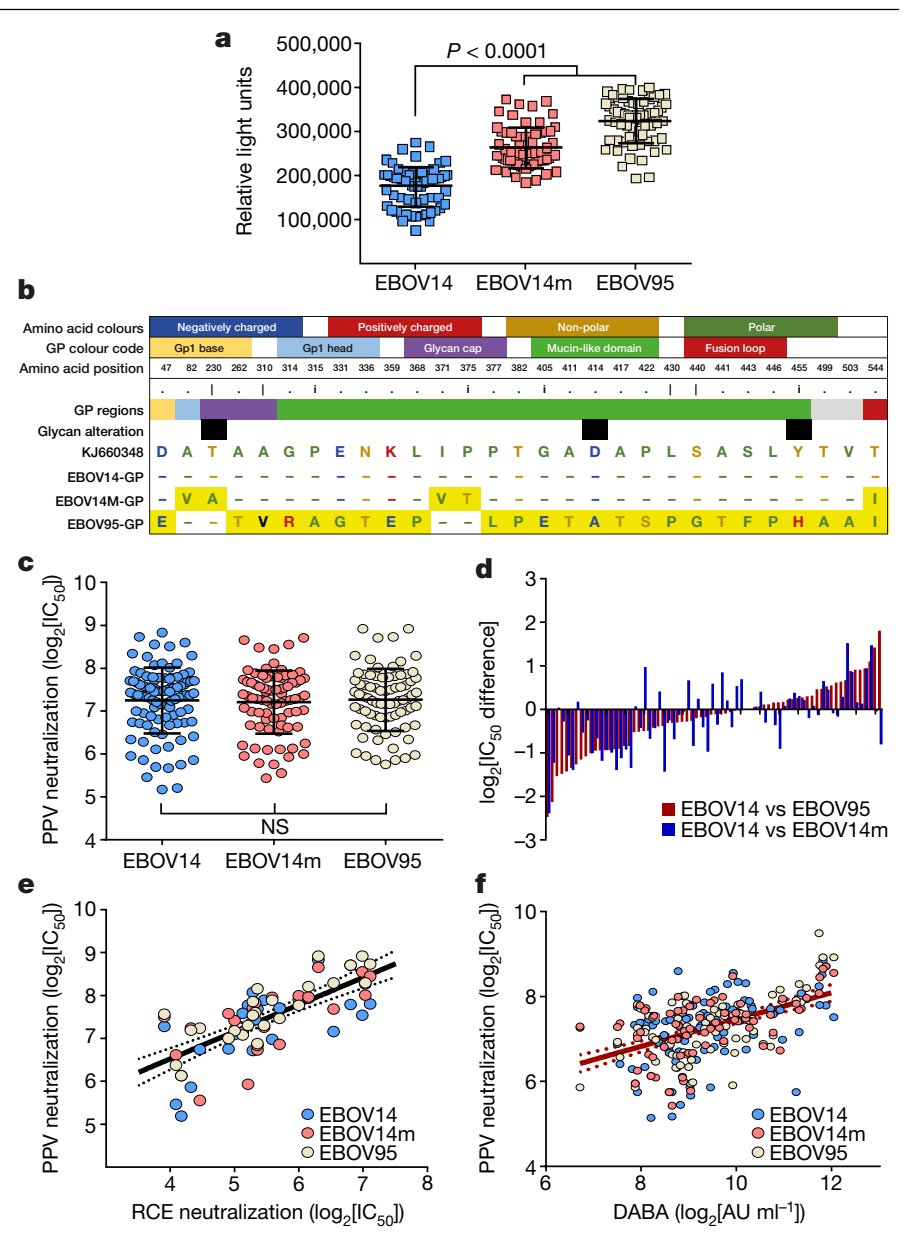

Fig. 1|EBOV-GP HIV-1 pseudo-typed virus neutralization assay. a, Virus produced in $10-\mathrm{cm}$ culture dishes $(n=60)$ using $285 \mathrm{ng}$ pEBOV14-GP, pEBOV14m or pEBOV95 plasmids. The infectivity of virus from each plate was assayed and plotted individually for the three virus strains (mean \pm s.d. of duplicate measurements; Kruskal-Wallis test).b, Amino acid differences in the glycoproteins (yellow boxes) of the three virus isolates studied. The differences are found in the GP1 base (orange), GP1 head (blue), glycan cap (purple), mucin-like domain (green) and fusion loop (red). Black bars indicate potential $N$-linked glycosylation site modifications. In pEBOV14m-GP, the glycosylation at the 230 position is lost. c, Neutralization potential of CPs against three virus strains (pEBOV14-GP, $n=98$; pEBOV95-GP, $n=80$; pEBOV14m-GP, $n=79$ ) expressed in $\mathrm{IC}_{50}$ (mean \pm s.d. of duplicate measurements; Kruskal-Wallis test). NS, not significant.d, Differences in IC ${ }_{50}$ neutralization titres between virus strain pairs by each post-recovery study participant. e, Positive association between PPV IC ${ }_{50}$ titres and the live virus plaque reduction neutralization test (PRNT).f, Positive association between PPV IC $_{50}$ neutralization titres and DABA. The simple linear regression is shown (solid line) with the $95 \%$ confidence interval (dotted line). AU, arbitrary units.

correlation than did neutralization versus DABA. The RCE and PPV assays target the same antibodies, whereas DABA measures all antibodies and some individuals would have differential responses (this was observed only in a very small number of donors).

Although nAbs are thought to develop later in infection ${ }^{28}$, our data demonstrate their presence as early as 30 days after the end of infection, consistent with previous studies ${ }^{29,30}$, which found that nAb levels are detectable and persist following viral clearance. Cross-sectional analysis of antibody responses did not indicate notable changes in titres during the observation window (about 500 days; Fig. 2a, b), comparable to the findings for neutralization of fully replicating virus (Fig. 2c). However, within individuals, sustained decline was often followed by a sharp increase in antibody titre (Fig. 2a-c). 

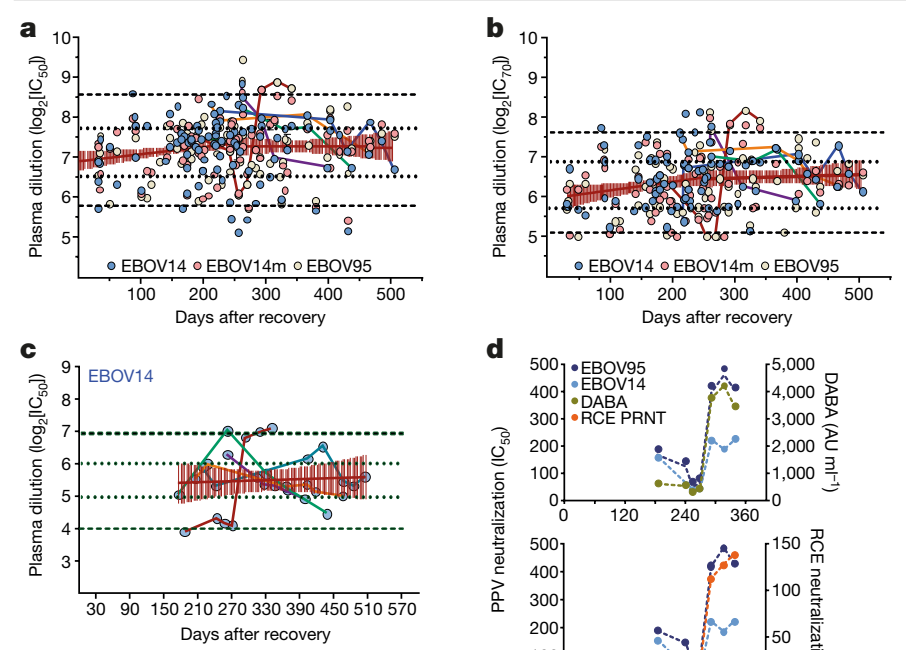

d
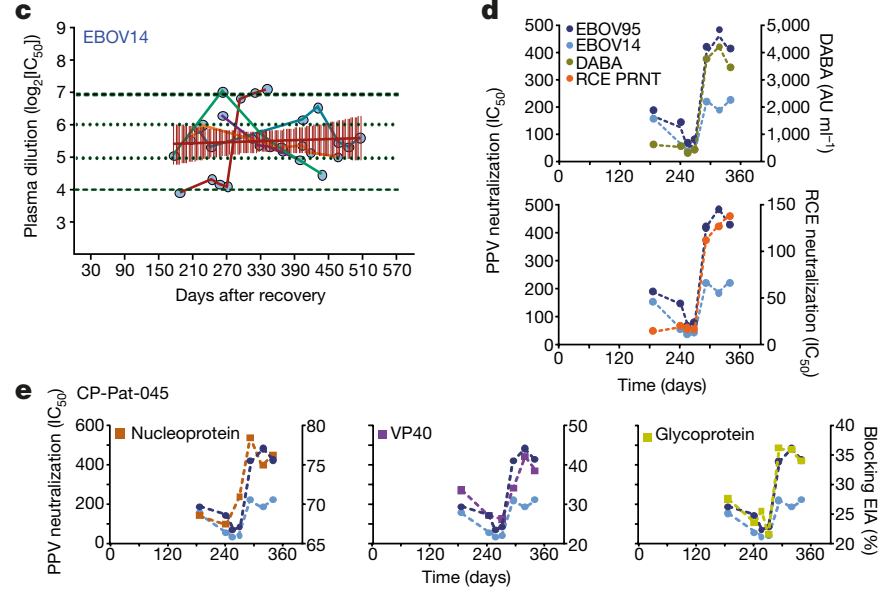

$\mathbf{f}$
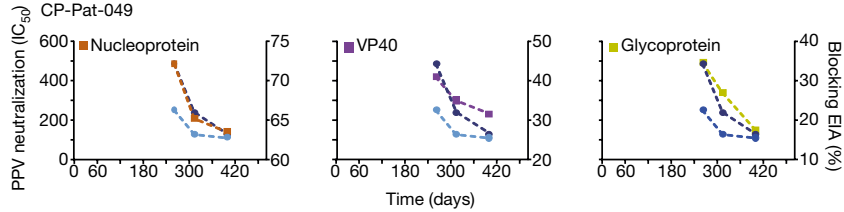

Fig. 2 | Convalescent plasma neutralizing antibody titres. a, b, nAb IC $\mathrm{C}_{50}(\mathbf{a})$ and $\mathrm{nAb} \mathrm{IC}_{70}(\mathbf{b})$ values against three PPV isolates (EBOV14 $(n=92)$, EBOV14 $\mathrm{m}$ $(n=70)$ and EBOV95 $(n=76))$ over time. $\mathbf{c}, \mathrm{nAb} \mathrm{IC}_{50}$ values against PRNT-EBOV14 $(n=30)$ over time. a-c, Day 0 is defined as the day when the virus PCR test became negative or when the individual was declared Ebola-free and discharged from the Ebola treatment unit. Individual lines indicate individuals who donated sequential plasma samples, demonstrating non-canonical antibody titre variation. Dotted lines, 25th-75th quartiles; dashed lines, 5th-95th quartiles; red vertical lines, $95 \%$ confidence intervals $(\mathrm{CI})$ of the linear association (red curve; calculated separately for each half of the observation period for PPV). d, Longitudinal post-cure antibody variation of donor CP-Pat-045 demonstrated by PPV neutralization of EBOV14 (light blue) and EBOV95 (dark blue) strains overlaid with virus neutralization using the RCE PRNT (green, top) or total antibodies measured by DABA (orange, bottom). e,f, Blocking EIAs using RCE were carried out to detect antibodies against the nucleoprotein (brown squares), the viral matrix protein 40 (VP40; purple squares) and the glycoprotein (green squares) using longitudinal plasma samples from donors CP-Pat-045 (e) and CP-Pat-049 (f).

The observed declines and subsequent rises in nAb levels identified in some of the study participants (Fig. 2a-c) indicates de novo antigen stimulation after recovery. This was comprehensively demonstrated in donor CP-Pat-045, whose antibody reactivity in all EIAs and including $n A b s$ initially decreased over a 45 -day period (sampled six months after recovery) before increasing suddenly over a 23-day period (Fig. 2d). It should be noted that all donors were tested for plasma EBOV RNA twice in Sierra Leone and were shown to be aviraemic before being discharged from Ebola treatment units. Furthermore, all samples received in the United Kingdom were subsequently re-tested upon arrival, and there was no detectable viraemia in the available samples taken in this observation period. Notably, the increase in antibody reactivity was higher against EBOV95-GP than EBOV14-GP, although participation in any vaccine study (where the immunogen would mimic the 1995 strain) was ruled out through self-reporting and later confirmed by the lead investigators of the two Ebola vaccine studies.

Following on from these observations, we tested samples from donor CP-Pat-045 and donors CP-Pat-018, -019, -021 and -049 using an additional panel of EIAs (targeting the EBOV glycoprotein, nucleoprotein and VP40 matrix protein) and found similar variations in antibody responses (Fig. 2e, f, Extended Data Fig. 4) indicating that antibody restimulation targeted viral antigens that are not present in current vaccines, and not just the glycoprotein. Furthermore, antibody responses showed similar variations in an IgG capture assay and a competitive antibody-binding immunoassay, both targeting the glycoprotein (Extended Data Fig. 5). Antibodies from donors CP-Pat-019 and -021 also increased before subsequently decreasing. The increases in EBOV antibodies from donor CP-Pat-045 occurred between mid-December 2015 and mid-January 2016. Two cases of Ebola were reported in mid-January 2016 in the northern districts, although Sierra Leone had been declared 'Ebola free' in November 2015 (https://www.theguardian.com/world/2015/ sep/04/sierra-leone-village-in-quarantine-after-ebola-death). These donors and a control group of donors who did not demonstrate late rises in $\mathrm{nAb}$ reactivity were interviewed. All denied any intercurrent illness, known exposure to individuals with Ebola or participation in EBOV vaccine studies. It should also be borne in mind that by definition these convalescent donors had to meet individually the Sierra Leone National Safe Blood criteria for fitness to donate blood. Furthermore, interviews and physical examinations were undertaken at each attendance for plasmapheresis. Although re-exposure to EBOV cannot be excluded, we assume that the increase in antibody reactivity represents de novo antigenic stimulation at immune-privileged sites, boosting immunity. The presence and ongoing replication of EBOV in such sites has been described as late clinical recrudescence and reporting of sporadic viral transmission ${ }^{31-36}$.

Given this high degree of intra-patient fluctuation in EBOV virus antibody responses, we used the available data to develop compartmental population pharmacodynamic models to quantify antibody stimulation and decay trends in this cohort. The strong association between nAbs and total antibody binding measured by DABA reactivity (Fig. 1f, Extended Data Fig. 1h) enabled us to use the more replete DABA dataset, which incorporates extensive longitudinal time-points (Supplementary Table 1f), to perform model selection for stimulation and decay trends. The best fitting models for stimulation and decay were objectively identified by comparison of the log-likelihood-based Akaike information criterion (AIC) and Bayesian information criterion (BIC) metrics (see Methods, Supplementary Table 3a-c) as a one-compartment model with reduced stimulation at high antibody levels (Fig. 3a) and a two-compartment decay model with saturable recycling of antibody (Fig. 3b). The rate constant for stimulation for total antibody binding reactivity was 0.03 per day, equivalent to a doubling time of 23 days (Supplementary Table 4), whereas the decay model provided a variable antibody concentration-dependent rate constant equivalent to 30 days at half the maximum antibody level measured (Supplementary Table 4). We then fitted the two best structural models, as selected using the DABA data, with the nAb titre values for the EBOV14-GP and EBOV95-GP strains and performed simulations (Fig. $3 \mathrm{e}-\mathrm{h}$ ). The calculated stimulation rate constants for the virus strain variants were 0.067 per day and 0.046 per day, respectively, possibly reflecting variation in epitope targets. The calculated endogenous nAb decay rates were similar for the different virus strains (0.025 per day for EBOV14-GP and 0.025 per day for EBOV95-GP) and matched the results initially found while modelling DABA reactivity (population mean of 0.028 per day; Supplementary Table 4). We calculated the resulting concentration-dependent half-lives at half maximal observed antibody levels as 51 and 70 days for EBOV14-GP and EBOV95-GP, respectively. Notably, our findings are congruent with recent studies that have modelled endogenous antibody metabolism ${ }^{37}$. To our knowledge, this is the first population model of antibody level dynamics in EBOV survivors. 


\section{a

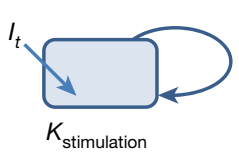

c
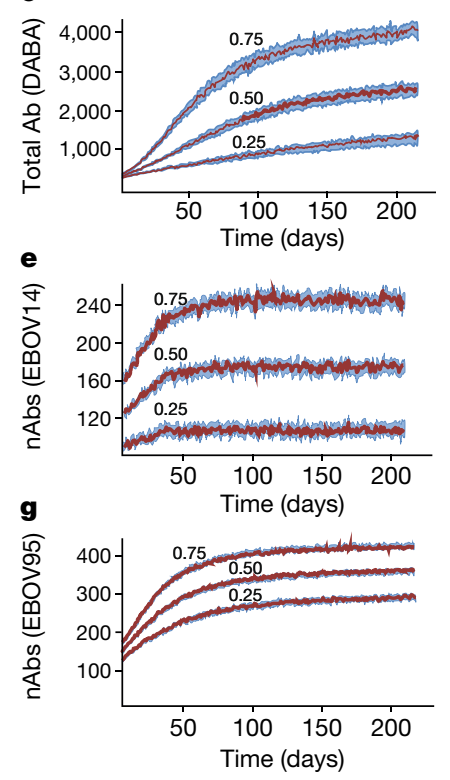

\section{d}
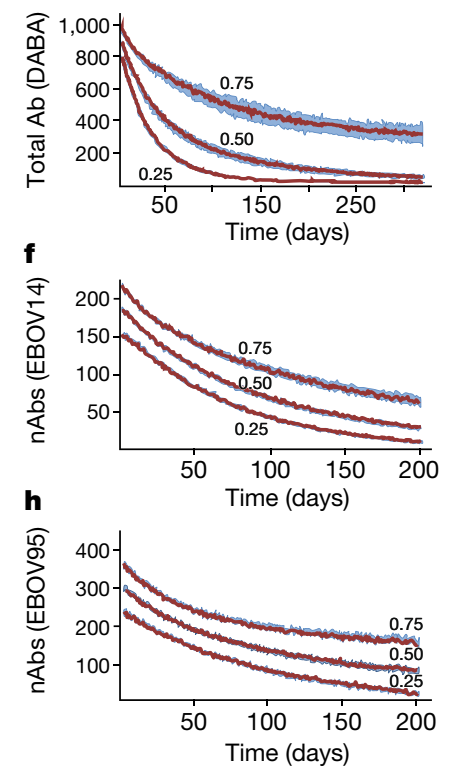

i

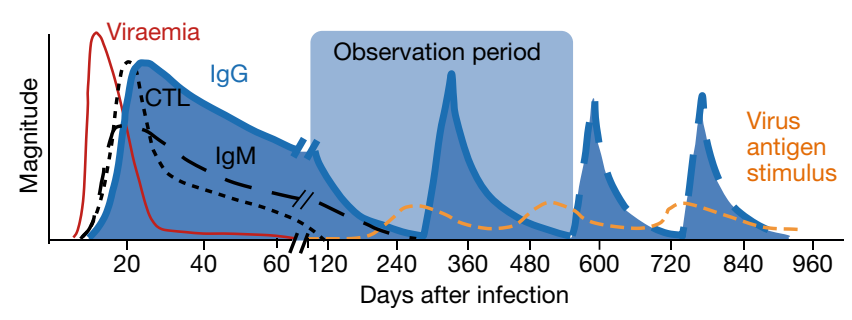

Fig. 3 | Rates of EBOV antibody decay and recovery following the 2013-2016 West Africa outbreak. a, Schematic depicting the one-compartment model for first-order stimulation (based on a logistic growth model). $I_{t}$, antibody level at time $t$ (the start of a stimulation or decay trend). b. Schematic depiction of the two-compartment decay and metabolism of IgG with saturable recycling. $k_{\text {end }}$, endogenous decay rate. $X_{1}, k_{\text {decay }}$ and $K_{\text {max }}$ denote antibody level in the compartment, the empirically observed antibody level-dependent rate constant and the maximal antibody level at which stimulation plateaus, respectively. $\mathbf{c}-\mathbf{h}$, Mean antibody stimulation (c, e, g) and decay $(\mathbf{d}, \mathbf{f}, \mathbf{h}) 25$ th, 50th and 75 th percentile concentrations. Shaded areas surrounding percentile trajectories indicate 0.05 and $0.95 \mathrm{CI}$. c, d, Total anti-EBOV reactivity as measured by DABA.e,f, Neutralizing antibody titres against the EBOV14 virus strain. g, h, Neutralizing antibody titres against the EBOV95 Kikwit strain. Percentiles are calculated stimulation or decay profiles from Monte Carlo simulations of a population of 1,000 randomly sampled individuals. i, Graphic illustration of the post-infection acquired immune responses, illustrating the virus antigen stimulation hypothesis extrapolated from simulation of median fitted parameter values from the selected models. It depicts the acute sharp post-infection increase and the slow decrease following recovery. The observation period during this study is highlighted, demonstrating the increase in antibody reactivity after what is predicted to be a new antigenic stimulation occurring below a threshold of antibody protection.

We next simulated the stimulation and decay profiles for 1,000 survivors of EBOV using the developed population models (Fig. $3 \mathrm{c}-\mathrm{h}$ ). The interquartile range of total antibody levels varied widely for the simulated cohort when tracked longitudinally, indicating a wide-ranging array of doubling times and half-lives. The mean simulated doubling times were 18.93 days (interquartile range: $11.68-33.62), 10.36$ days
(9.96-10.81) and 13.76 days (9.52-23.56), for total binding antibody, nAbs against EBOV14-GP and nAbs against EBOV95-GP, respectively, indicating that overall EBOV14-GP was stimulated most quickly and with the least variability, which is reasonable given that this was the 2013-2016 epidemic strain. The median simulated endogenous decay half-lives were 20.86 days (3.81-42.81), 27.40 days (19.62-41.66) and 28.23 days (24.33-33.33), respectively, consistent with previous estimates of IgG half-life ${ }^{37}$.

Increasing antibody reactivity was shown in a high proportion of study participants, somewhere between 200 and 300 days after recovery, by longitudinal analysis of DABA (Fig. 2e, Extended Data Fig. 6), blocking EIA (Fig. 2e, Extended Data Fig. 4), IgG capture and competitive EIA (Extended Data Fig. 5), as well as with antibody neutralization measurements (Fig. 2d). This suggests that as antibody responses are waning, antigen levels increase, resulting in a boost to the residual primary antibody response. When we compared the lowest observed antibody titres after decline with the highest antibody titres following stimulation (before further de novo decline), we observed a statistical difference in antibody levels $(n=18, P<0.0014$; Extended Data Fig. 7$)$. We have simulated a typical decay-restimulation-decay profile based on population median parameters and starting levels, demonstrating a projected typical scenario in a substantial proportion of EBOV survivors (Fig. 3i).

Analyses of naturally occurring nAb responses in our Ebola- $\mathrm{CP}$ donor cohort revealed a high degree of variation in the strength and breadth of induced responses. Longitudinal analysis of B cell responses in EBOV-infected individuals has revealed stark changes in immunoglobulin subclass switching, heightened alterations to hypermutations and naive $B$ cell restimulations over time ${ }^{38}$. Our results suggest that EBOV antigen re-exposure contributes to these observed alterations in antibody phenotypes. It is encouraging to find strong neutralization cross-reactivity between EBOV strains representing outbreaks 20 years apart. This provides confidence that antibodies induced either through natural infection or via a vaccine should provide protection against future outbreaks. Our results indicate that the evolution of $\mathrm{EBOV}^{39,40}$, albeit slow, may result in altered neutralizing potential and therefore loss of vaccine efficacy (Fig. 1c, Extended Data Fig. 1f). Furthermore, if $\mathrm{CP}$ with broadly neutralizing activity were to be used in therapeutic protocols, then combining plasmas from several individuals may ensure a more successful outcome. The best option could be the preparation of a hyperimmune intravenous immunoglobulin blood product from a panel of donors, rather than relying on the use of individually sourced components as at present. The high frequency of de novo antigenic stimulation described within our cohort indicates a need for heightened surveillance of survivors to meet the potential clinical needs associated with virus recrudescence. Subclinical recrudescence may intensify the long-lasting post-Ebola sequelae suffered by most EBOV survivors ${ }^{41,42}$. The cohort of CP donors studied here, however, represents a highly selected group of healthy individuals, further chosen through the use of field testing to have plasma antibodies to EBOV in the upper quartiles of serological reactivity ${ }^{21}$. Therefore, they may represent the convalescent individuals who are least likely to suffer viral recrudescence. Occult virus persistence is therefore likely to be more frequent than previously predicted, supporting findings that the virus persists at sequestered sites in some individuals ${ }^{2,43}$. In a case study of an immunocompromised individual infected with HIV-1 (CD4 cell count $\left.46 \mathrm{\mu l}^{-1}\right)$, EBOV was detected in semen two years after the individual was discharged from the treatment unit ${ }^{37}$, further underlining the importance of immune-competence for EBOV clearance. A longer and more frequent sampling would provide a more accurate indication of the extent of antibody restimulation in these survivors of Ebola.

The calculated mean half-life at median antibody levels allowed us to predict the time taken to reach $95 \%$ depletion of any given level after antigenic stimulation; given the exponential decay rate, we predict that the duration of six half-life periods (about 180-417 days) will result in 


\section{Article}

depletion of antibody levels by more than $95 \%$. As a result, protection of EBOV survivors from viral recrudescence mediated by acquired immunity is likely to last for $0.5-2$ years after recovery unless boosted.

Continued surveillance of EBOV survivors is warranted, considering the frequency of sub-clinical de novo antigenic stimulation we have described. Vaccination could be considered to boost protective antibody responses in survivors. This would also have a particular role if EBOV survivors are to be considered as plasma donors for use in future anti-Ebola passive immunotherapy.

\section{Online content}

Any methods, additional references, Nature Research reporting summaries, source data, extended data, supplementary information, acknowledgements, peer review information; details of author contributions and competing interests; and statements of data and code availability are available at https://doi.org/10.1038/s41586-020-03146-y.

1. Kasangye, K. A. et al. in Advances in Ebola Control (ed. Okware, S. I.) (IntechOpen, 2017)

2. Heeney, J. L. Ebola: hidden reservoirs. Nature 527, 453-455 (2015).

3. Sullivan, N. J., Martin, J. E., Graham, B. S. \& Nabel, G. J. Correlates of protective immunity for Ebola vaccines: implications for regulatory approval by the animal rule. Nat. Rev. Microbiol. 7, 393-400 (2009).

4. Saphire, E. O. et al. Systematic analysis of monoclonal antibodies against Ebola virus GP defines features that contribute to protection. Cell 174, 938-952.e13 (2018).

5. Henao-Restrepo, A. M. et al. Efficacy and effectiveness of an rVSV-vectored vaccine expressing Ebola surface glycoprotein: interim results from the Guinea ring vaccination cluster-randomised trial. Lancet 386, 857-866 (2015)

6. Henao-Restrepo, A. M. et al. Efficacy and effectiveness of an rVSV-vectored vaccine in preventing Ebola virus disease: final results from the Guinea ring vaccination, open-label, cluster-randomised trial (Ebola Ca Suffit!). Lancet 389, 505-518 (2017).

7. Zhu, F. C. et al. Safety and immunogenicity of a recombinant adenovirus type-5 vector-based Ebola vaccine in healthy adults in Sierra Leone: a single-centre randomised, double-blind, placebo-controlled, phase 2 trial. Lancet 389, 621-628 (2017).

8. Ringe, R. \& Bhattacharya, J. Preventive and therapeutic applications of neutralizing antibodies to human immunodeficiency virus type 1 (HIV-1). Ther. Adv. Vaccines 1, 67-80 (2013).

9. VanBlargan, L. A., Goo, L. \& Pierson, T. C. Deconstructing the antiviral neutralizing-antibody response: implications for vaccine development and immunity. Microbiol. Mol. Biol. Rev. 80, 989-1010 (2016).

10. Crowe, J. E. Jr. Principles of broad and potent antiviral human antibodies: insights for vaccine design. Cell Host Microbe 22, 193-206 (2017).

11. Garraud, O. Use of convalescent plasma in Ebola virus infection. Transfus. Apher. Sci. $\mathbf{5 6}$ 31-34 (2017)

12. Zhang, Q. et al. Potent neutralizing monoclonal antibodies against Ebola virus infection. Sci. Rep. 6, 25856 (2016).

13. King, L. B., West, B. R., Schendel S. L. \& Saphire, E. O. The structural basis for filovirus neutralization by monoclonal antibodies. Curr. Opin. Immunol. 53, 196-202 (2018).

14. Gaudinski, M. R. et al. Safety, tolerability, pharmacokinetics, and immunogenicity of the therapeutic monoclonal antibody mAb114 targeting Ebola virus glycoprotein (VRC 608): an open-label phase 1 study. Lancet 393, 889-898 (2019).

15. van Griensven, J., Edwards, T. \& Baize, S. Efficacy of convalescent plasma in relation to dose of Ebola virus antibodies. N. Engl. J. Med. 375, 2307-2309 (2016).

16. van Griensven, J. et al. The use of Ebola convalescent plasma to treat Ebola virus disease in resource-constrained settings: a perspective from the field. Clin. Infect. Dis. 62, 69-74 (2016).

17. Chippaux, J. P., Boyer, L. V. \& Alagón, A. Post-exposure treatment of Ebola virus using passive immunotherapy: proposal for a new strategy. J. Venom. Anim. Toxins Incl. Trop. Dis. 21, 3 (2015).

18. Wec, A. Z. et al. Development of a human antibody cocktail that deploys multiple functions to confer pan-Ebolavirus protection. Cell Host Microbe 25, 39-48.e5 (2019).

19. Flyak, A. I. et al. Cross-reactive and potent neutralizing antibody responses in human survivors of natural Ebolavirus infection. Cell 164, 392-405 (2016).

20. Bramble, M. S. et al. Pan-filovirus serum neutralizing antibodies in a subset of Congolese Ebolavirus infection survivors. J. Infect. Dis. 218, 1929-1936 (2018).
21. Tedder, R. S. et al. Detection, characterization, and enrollment of donors of Ebola convalescent plasma in Sierra Leone. Transfusion 58, 1289-1298 (2018).

22. Baize, S. et al. Emergence of Zaire Ebola virus disease in Guinea. N. Engl. J. Med. 371, 1418-1425 (2014).

23. Carroll, S. A. et al. Molecular evolution of viruses of the family Filoviridae based on 97 whole-genome sequences. J. Virol. 87, 2608-2616 (2013).

24. Hoffmann, M. et al. A polymorphism within the internal fusion loop of the Ebola virus glycoprotein modulates host cell entry. J. Virol. 91, e00177-17 (2017).

25. Urbanowicz, R. A. et al. Human adaptation of Ebola virus during the West African outbreak. Cell 167, 1079-1087.e5 (2016)

26. Marzi, A. et al. Recently identified mutations in the Ebola virus-Makona genome do not alter pathogenicity in animal models. Cell Rep. 23, 1806-1816 (2018).

27. Wilkinson, D. E. et al. Comparison of platform technologies for assaying antibody to Ebola virus. Vaccine 35, 1347-1352 (2017).

28. Williamson, L. E. et al. Early human B cell response to Ebola virus in four U.S. survivors of infection. J. Virol. 93, e01439-18 (2019).

29. Rimoin, A. W. et al. Ebola virus neutralizing antibodies detectable in survivors of the Yambuku, Zaire outbreak 40 years after infection. J. Infect. Dis. 217, 223-231 (2018).

30. Brown, J. F. et al. Anti-Ebola virus antibody levels in convalescent plasma and viral load after plasma infusion in patients with Ebola virus disease. J. Infect. Dis. 218, 555-562 (2018).

31. Dokubo, E. K. et al. Persistence of Ebola virus after the end of widespread transmission in Liberia: an outbreak report. Lancet Infect. Dis. 18, 1015-1024 (2018).

32. Subissi, L. et al. Ebola virus transmission caused by persistently infected survivors of the 2014-2016 outbreak in West Africa. J. Infect. Dis. 218 (Suppl. 5), S287-S291 (2018).

33. Shantha, J. G. et al. Ebola virus persistence in ocular tissues and fluids (EVICT) Study: reverse transcription-polymerase chain reaction and cataract surgery outcomes of Ebola survivors in Sierra Leone. EBioMedicine 30, 217-224 (2018).

34. Whitmer, S. L. M. et al. Active Ebola virus replication and heterogeneous evolutionary rates in EVD survivors. Cell Rep. 22, 1159-1168 (2018).

35. Den Boon, S. et al. Ebola virus infection associated with transmission from survivors. Emerg. Infect. Dis. 25, 249-255 (2019).

36. Purpura, L. J. et al. Ebola virus RNA in semen from an HIV-positive survivor of Ebola. Emerg. Infect. Dis. 23, 714-715 (2017).

37. Kendrick, F. et al. Analysis of a compartmental model of endogenous immunoglobulin $G$ metabolism with application to multiple myeloma. Front. Physiol. 8, 149 (2017).

38. Davis, C. W. et al. Longitudinal analysis of the human B cell response to Ebola virus infection. Cell 177, 1566-1582.e17 (2019)

39. Carroll, M. W. et al. Temporal and spatial analysis of the 2014-2015 Ebola virus outbreak in West Africa. Nature 524, 97-101 (2015).

40. Dudas, G. et al. Virus genomes reveal factors that spread and sustained the Ebola epidemic. Nature 544, 309-315 (2017).

41. Scott, J. T. et al. Post-Ebola syndrome, Sierra Leone. Emerg. Infect. Dis. 22, 641-646 (2016).

42. Jagadesh, S. et al. Disability among Ebola survivors and their close contacts in Sierra Leone: a retrospective case-controlled cohort study. Clin. Infect. Dis. 66, 131-133 (2018).

43. Jacobs, M. et al. Late Ebola virus relapse causing meningoencephalitis: a case report. Lancet 388, 498-503 (2016).

Publisher's note Springer Nature remains neutral with regard to jurisdictional claims in published maps and institutional affiliations.

(c) The Author(s), under exclusive licence to Springer Nature Limited 2021

The Ebola-CP Consortium

M. G. Semple ${ }^{2,3}$, J. T. Scott ${ }^{2,3,4}$, S. M. Gevao ${ }^{12,17}$, F. Sahr ${ }^{17,18}$, C. P. Cole ${ }^{11,30}$, J. Russell ${ }^{17}$, S. Baker ${ }^{12,34}$, O. Kargbo ${ }^{12}$, P. Kamara ${ }^{12}$, M. Lado $^{20}$, C. S. Brown ${ }^{20}$, B. Conton ${ }^{21}$, J. van Griensven ${ }^{15}$, R. Ravinetto ${ }^{15}$, Y. Claeys ${ }^{15}$, R. S. Tedder ${ }^{2,7,831}$, R. Gopal ${ }^{6}$, T. J. G. Brooks ${ }^{6}$, C. C. Smith ${ }^{3,10,29}$, H. A. Doughty ${ }^{13,14}$, A. Mari Saez ${ }^{22}$, A. H. Kelly ${ }^{23}$, J. K. Baillie ${ }^{24}$, N. Shindo ${ }^{25}$ D. Pfeifer ${ }^{25}$, D. L. Hoover ${ }^{26}$, W. A. Fischer $I^{27}{ }^{27}$ D. A. Wohl ${ }^{27}$, N. M. Thielman ${ }^{28}$, P. W. Horby ${ }^{16}$ L. Merson ${ }^{16}$, P. G. Smith ${ }^{9}$ \& T. Edwards ${ }^{9}$

${ }^{20}$ King's Sierra Leone Health Partnership, King's Health Partners and King's College London London, UK. ${ }^{21}$ Physio Fitness Rehabilitation Centre, Freetown, Sierra Leone. ${ }^{22}$ Institute for Tropical Medicine and International Health, Charité, Berlin, Germany. ${ }^{23}$ Department of Sociology, Philosophy and Anthropology, University of Exeter, Exeter, UK. ${ }^{24}$ The Roslin Institute, University of Edinburgh, Edinburgh, UK. ${ }^{25}$ Department of Pandemic and Epidemic Diseases, World Health Organization, Geneva, Switzerland. ${ }^{26} \mathrm{ClinicalRM}$ Inc, Hinckley, $\mathrm{OH}$ USA. ${ }^{27}$ Department of Medicine, University of North Carolina, Chapel Hill, NC, USA. ${ }^{28}$ Duke University School of Medicine, Durham, NC, USA. ${ }^{34}$ Deceased: S. Baker. 


\section{Methods}

\section{Ebola survivor cohort}

Ebola virus disease survivors $(n=115)$, previously described ${ }^{42}$ with certificates (issued by Ebola treatment centres on discharge) were recruited as potential donors through 34 Military Hospital, Freetown, and the Sierra Leone Association of Ebola Survivors as participants in the study 'Convalescent plasma (CP) for early Ebola virus disease in Sierra Leone'. The study (ISRCTN13990511 \& ACTR201602001355272) was approved by the Scientific Review Committee and Sierra Leone Ethics, authorized by the Pharmacy Board of Sierra Leone (PBSL/CTAN/ MOHSCST001) and sponsored by the University of Liverpool. All participants provided written consent for data collected in this study.

Volunteers were considered suitable to donate plasma if they tested negative for blood-borne infections (hepatitis B, hepatitis C, HIV, malaria and syphilis), had had two documented negative EBOV PCR tests $72 \mathrm{~h}$ apart, had no acute febrile illness and had no comorbidity, such as heart failure, to suggest that they might be at increased risk of adverse events during apheresis. Volunteers were not excluded if they exhibited indications of post-Ebola syndrome (PES; for example, musculoskeletal pain, headache or ocular problems), although such complaints were noted and subsequently contributed to the characterization of PES ${ }^{41,42,44}$. The majority of the participants were male $(n=82)$, their age ranging between 18 and 52 with a median of 27 years old. The female $(n=33)$ age range was between 18 and 42 with a median of 27 years old (Supplementary Table 1a).

For transfusion safety reasons, donor identity numbers were not confidential to donors during the conduct of the study; for the avoidance of doubt, donor identity numbers have since been dissociated. All participants $(n=115)$ were tested using DABA, blocking EIA and IgG capture immunoassays ${ }^{21}$. PPV antibody neutralization assays were performed with a subset of participants not selected on any criteria other than sample availability $(n=52)$. The compartmental population pharmacodynamics model was developed on the more replete DABA dataset using those participants with longitudinal data $(n=51)$ (Supplementary Table 1f).

\section{Cell culture}

HEK293T (ATCC CRL-3216) and TZM-bl ${ }^{45-49}$ (acquired from NHI AIDS Reagent Program) cells are adherent cell lines cultivated in Dulbecco's modified Eagle's medium (Invitrogen:12491-023), supplemented with $10 \%$ heat-treated fetal bovine serum (FBS) (Sigma: F7524), $2 \mathrm{mM} / \mathrm{ml}$ L-glutamine (Invitrogen: 25030024), $100 \mathrm{U} / \mathrm{ml}$ penicillin (Invitrogen: 15140148) and $100 \mathrm{mg} / \mathrm{ml}$ streptomycin (Invitrogen:15140148), referred to as complete DMEM (Thermo Fisher:12491023). Cells were grown in a humidified atmosphere at $37^{\circ} \mathrm{C}$ and $5 \% \mathrm{CO}_{2}$. Vero E6 cells (ECACC: 85020206) were grown in VP-SFM (Thermo Fisher:11681-020). All cell lines were tested monthly for mycoplasma contamination.

\section{EBOV PPV construct design}

Three viral strain glycoprotein genes were cloned into pCDNA3.1 produced by GeneArt using gene synthesis: a 2014 isolate (KP096421)2, a variant carrying the $\mathrm{A} 82 \mathrm{~V}, \mathrm{~T} 230 \mathrm{~A}, \mathrm{I} 371 \mathrm{~V}, \mathrm{P} 375 \mathrm{~T}$, and T544I mutations (Fig. 1b) identified by analysis of sequenced EBOV strains between March and August $2014^{39}$ and the AY3544581995 Kikwit isolate ${ }^{50}$. The latter was used in ring vaccinations during the 2014 epidemic.

\section{EBOV PPV production}

We chose to utilize the HIV-1SG3 $\Delta$ Env and EBOV-GP expression plasmids, co-transfected into HEK293T cells, to generate infectious PPV stocks $^{47,51,52}$. The EBOV-GP-pseudotyped lentiviral system generates single-cycle infectious viral particles. HEK293T cells were plated at a density of $1.2 \times 10^{6}$ in a $10-\mathrm{cm}$ diameter tissue culture dish (Corning: 430167 ) in $8 \mathrm{ml}$ complete DMEM and incubated overnight. The cells were transfected with $2 \mu \mathrm{g}$ pSG $3 \Delta \mathrm{env}$ along with $0.285 \mu \mathrm{g}$ of a plasmid expressing EBOV-GP using a cationic polymer transfection reagent (Polyethylenimine, Polysciences: 23966-2), in the presence of OptiMEM (Invitrogen: 31985-070). OptiMEM was replaced $6 \mathrm{~h}$ after transfection with $8 \mathrm{ml}$ complete DMEM. Seventy-two hours after transfection, supernatant containing the generated stock of single-cycle infectious EBOV-GP pseudotyped virus particles was harvested, passed through a $0.45-\mu \mathrm{M}$ filter and stored in aliquots at $-80^{\circ} \mathrm{C}$. EBOV-GP plasmid $(285$ ng per 10-cm culture dish) was used to produce a large virus stock that was tested for infectivity (Fig. 1a) then pooled, aliquoted and stored at $-80^{\circ} \mathrm{C}$.

\section{EBOV PPV infection}

EBOV infectivity was determined through infection of TZM-bl cell lines where luciferase activity (expressed from LTR promoter) is under the control of Tat expressed from the HIV-1 backbone. We used $100 \mu \mathrm{l}$ EBOV-GP virus to infect $1.5 \times 10^{4} \mathrm{TZM}$-bl cells per well for $6 \mathrm{~h}$ in a white 96-well plate (Corning: CLS3595). Following infection, $150 \mu \mathrm{l}$ per well DMEM complete was added to the cells. Forty-eight hours after infection, medium was discarded from the wells, cells were washed with phosphate-buffered saline (PBS, ThermoFisher:12899712) and lysed with $30 \mu \mathrm{l}$ cell lysis buffer (Promega: E1531), and luciferase activity was determined by luciferase assay (Promega: E1501) using a BMGLabtech FluoroStar Omega luminometer. Negative controls included pseudotyped virus bearing no glycoproteins and TZM-bl cells alone, which routinely resulted in luminescence of 3,000-7,000 relative light units (RLU).

\section{EBOV PPV neutralization}

Plasma samples $(n=52)$ from Ebola convalescent plasma and healthy blood donors $(n=6)$ were heat treated at $56^{\circ} \mathrm{C}$ for $30 \mathrm{~min}$ and centrifuged for $15 \mathrm{~min}$ at $13,000 \mathrm{RPM}$. Aliquots were then stored at $-80^{\circ} \mathrm{C}$. Plasma samples were serially diluted $50 \%$ with complete DMEM; $13 \mu \mathrm{l}$ plasma dilution was incubated with $200 \mu \mathrm{lEBOV}$-GP PPV for $1 \mathrm{~h}$ at room temperature. We used $100 \mu$ l of virus/plasma dilution to infect TZM-bl cells as described above. Luciferase activity readings of neutralized virus were analysed (i) by considering $0 \%$ inhibition as the infection value of the virus in the absence of convalescent plasma included in each experiment, (ii) by considering $0 \%$ inhibition as the infection value of two consecutive high dilutions that did not inhibit virus entry. Both methods produced highly correlated results (Extended Data Fig. 2d) and the latter was used. The neutralization potential of a CP was represented as the plasma dilution that reduced viral infectivity by $50 \%$ $\left(\mathrm{IC}_{50}\right)$ or by $70 \%\left(\mathrm{IC}_{70}\right)$.

\section{Enzyme immune assays}

HIV-1-P24 capsid. Samples were diluted in 0.1\% Empigen (Sigma: 30326) in TBS before the ELISA assay (Fisher: 10167481). The p24 assays were conducted using the Aalto Bio Reagents Ltd protocol and recombinant p24 standard, p24 coating antibody (polyclonal sheep anti-HIV-1-p24 gag, Aalto Bio Reagents Ltd: D7320), secondary conjugate (alkaline phosphatase conjugate of mouse monoclonal anti-HIV-1-p24, Boehringer Mannheim:1089-161) and ELISA light assay buffer. Plates were incubated for $30 \mathrm{~min}$ at room temperature before we measured luminescence with a FLUOStar Omega luminometer (BMG LabTech).

Double antigen bridging assay (DABA). We measured EBOV GP targeting antibody present in Ebola survivor CP samples. EBOV GP antigen, Mayinga Zaire EBOV strain (IBT Bioservices: 0501-016) was pre-coated onto the 'solid phase', while a second antigen conjugated to horseradish peroxidase (HRP) acted as the detector, binding to EBOV antibodies captured on the solid-phase antigen in the first incubation step. Antibody reactivity was expressed as arbitrary units per $\mathrm{ml}(\mathrm{AU} / \mathrm{ml})$ as compared to a standard comprising five reactive donor samples that were pooled and set as $1,000 \mathrm{AU} / \mathrm{ml}^{21}$. 
Blocking EIA. Antibody levels in CP to EBOV GP (glycoprotein), VP40 and NP (nucleoprotein) were determined by blocking of the binding of specific rabbit EBOV anti-peptide (GP, VP40, NP) antibodies (IBT Bioservices) to EBOV Makona virion-coated microplates. Microplate wells were coated with a 10,000-fold dilution of concentrated Ebola virions. EBOV patient $C P$ and negative control $C P$ dilutions $(1 / 100)$ were reacted on virion-coated microplates for 4-6h. CP dilutions were removed and plates were then reacted with EBOV anti-peptide antibodies. Bound rabbit antibodies were detected by species-specific horseradish peroxidase conjugate (DAKO:P03991-2). Evidence of EBOV protein-specific human antibodies in $\mathrm{CP}$ was determined by blocking of the binding of the antipeptide antibody compared to the blocking of binding by the $\mathrm{CP}$ negative control. Results were expressed as a percentage of blocking of the $\mathrm{CP}$ negative control reactivity.

IgG capture assay. IgG antibodies present in CP were captured onto a solid phase coated with rabbit hyperimmune anti-human $\gamma$-Fc and interrogated in a second incubation with HRP-conjugated EBOV GP as above. Reactivity was expressed as binding ratios derived as sample $\mathrm{OD} /$ cut-off $\mathrm{OD}^{21}$.

\section{Plaque reduction neutralization test}

The wild-type strain used for assays was EBOV Makona (GenBank accession number KJ660347 ${ }^{21}$, isolated from a female Guinean patient in March 2014 (virus provided to PHE Porton by S. Günther, Bernhard-Nocht-Institute for Tropical Medicine, Hamburg, Germany). The virus was propagated in Vero E6 cells and culture supernatant virions were concentrated by ultracentrifugation through a $20 \%$ glycerol cushion; pellets were resuspended in sterile PBS at a titre of $10^{9}$ focus-forming units (FFU) per $\mathrm{ml}$.

The wild-type virus neutralizing antibody titre in $\mathrm{CP}$ was determined by reacting serial dilutions of $C P$ with 100 FFU of EBOV virions for $1 \mathrm{~h}$ at room temperature to allow antibody binding. The EBOV virion CP mixture was adsorbed to Vero E6 monolayers for $1 \mathrm{~h}$ and then overlaid with cell growth medium containing $1 \%(\mathrm{v} / \mathrm{v})$ Avicel (Sigma-Aldrich). After 80-90 h, EBOV foci were visualized by immunostaining with anti-VLP (Zaire EBOV) antibodies (IBT Bioservices). All work was undertaken under ACDP containment level 4 conditions.

\section{EBOV antibody decay and restimulation modelling}

Compartmental population analysis was performed to model the stimulation and decay of antibody levels. All modelling and simulations were performed using Pmetrics version $1.4^{53}$ within $\mathrm{R}$ version 3.2.2 $2^{54}$. Antibody levels of EBOV survivors were sampled at different number of instances, at varying intervals post convalescence due to limitations of follow-up adherence in the field. Different parts of decay-stimulation profiles were therefore captured, with only a few instances of contiguous decaystimulation or stimulation-decay profiles being captured.Stimulation and decay data were therefore modelled separately to most efficiently use the data. Antibody stimulation-decay trends with 2 or more data points were included in population analysis as this methodology has been proven to maximally use sparse clinical data for drug development ${ }^{55,56}$. All points were plotted and visualized. An 'ascend' or a 'descend' was defined according to the prevailing trend. A 20\% alteration in direction was tolerated as part of the prevailing ascend or descend as appropriate.

\section{Structural model}

Structural model selection was performed for the most replete DABA data set. Model fitting and selection were performed using previously published protocols for fitting clinical data sets as described below ${ }^{57,58}$. In brief, linear regression (intercept close to 0 , slope close to 1 ) was used to assess the goodness-of-fit of the observed-predicted values, the coefficient of determination of the linear regression and minimization of log-likelihood, AIC and BIC values were used for model selection.
A change in BIC drop of more than 2 is generally considered to be significant; with 2-6 indicating positive-to-strong evidence, 6-10 indicating strong evidence and $>10$ indicating very strong evidence ${ }^{59}$.

Further details of this analysis leading to the choice of models and analysis of the fit of models to data can be found in Supplementary Tables 3, 4 and Extended Data Figs. 8, 9.

All chosen structural models showed strong-to-very-strong evidence of describing the data the best out of the compared models. Two structural models were tested for antibody stimulation, a one-compartmental stimulation model and a one-compartmental model with saturable stimulation, based on the logistic growth model. The logistic growth model framework allows for plateauing antibody levels, as observed for a subset of stimulation profiles. For antibody decay, four structural models were tested; a one-compartment decay model with first order elimination, a two-compartment decay model with first order elimination from the central compartment, and the above two structural models with saturable recycling offsetting the endogenous elimination rate.

Antibody stimulation was best modelled using the onecompartmental model with saturable stimulation as described by equation (1):

$$
\frac{\mathrm{d} X_{1}}{\mathrm{~d} t}=k_{\text {growth }} X_{1}\left(1-\frac{X_{1}}{K_{\max }}\right)
$$

where $X_{1}, k_{\text {growth }}$ and $K_{\max }$ denote antibody level in the compartment, the first order rate constant for endogenous antibody stimulation and the maximal antibody level at which stimulation plateaus, respectively.

For antibody decay, the two-compartment decay model with saturable FcRn-dependent recycling (equations (2-4)) as used to model antibody decay in multiple laboratory studies ${ }^{37}$ was found to best describe the data.

$$
\begin{gathered}
\frac{\mathrm{d} X_{1}}{\mathrm{~d} t}=-k_{\mathrm{decay}} X_{1}-k_{\mathrm{cp}} X_{1}+k_{\mathrm{pc}} X_{2} \\
\frac{\mathrm{d} X_{2}}{\mathrm{~d} t}=k_{\mathrm{cp}} X_{1}-k_{\mathrm{pc}} X_{2} \\
k_{\text {decay }}=k_{\text {end }}-\left(\frac{V_{\max }}{X_{1}+K_{\mathrm{m}}}\right)
\end{gathered}
$$

where $X_{1}$ and $X_{2}$ are the antibody levels in the central and peripheral compartments. The rate constants $k_{\text {decay }}, k_{\mathrm{cp}}$ and $k_{\mathrm{pc}}$ denote the empirically observed antibody level dependent rate constant and the first order rate constants to and from the peripheral compartment, respectively. $k_{\text {decay }}$ is in turn dependent on the endogenous decay rate $k_{\text {end }}$, which is offset by an antibody-dependent saturable recycling rate described by a Michaelis-Menten term with parameters $V_{\max }$ and $K_{\mathrm{m}}$ denoting the maximal recycling rate and antibody level at which half the maximal recycling rate occurs, respectively. The optimal structural models above were then used to model the more sparse nAb assay datasets, allowing for comparability between DABA and nAb model parameters. Generally, individual predicted versus observed value correlations were excellent $\left(R^{2}>0.8\right)$ and population predictions versus observed values were good $\left(R^{2}>0.6\right)$.

Monte Carlo simulations were performed using Pmetrics as previously described ${ }^{57,58}$. In brief, 1,000 individuals were randomly sampled from parameter distributions defined in the population models of antibody stimulation and decay. The interquartile range of modelled antibody levels was then plotted longitudinally for average starting antibody levels for decay and stimulation profiles (Fig. 3c-h).

With regard to the choice to model the stimulation and decay data separately: in principle, an immune response followed by a gradual return to baseline post-stimulus could be characterized by a single 
pharmacodynamic model. In the simplest form, the dynamics can be described by a single-compartment model with the stimulus placed on the input rate and first order elimination, although more mechanistic models based on known pharmacology may also be appropriate if the data are of sufficient quality to estimate the unknown model components. In a controlled trial setting, the onset of a stimulus event would be controlled and the subsequent immune response measured relative to this origin with sufficient frequency to capture the dynamics over time. By contrast, this study was observational with plasma samples taken intermittently that captured only part of the changing levels in the nAbs-either the growth or decay phase in most cases, but on occasion both. Given the lack of detectable viral load and the observational nature of the nAb response data, the ability to fit a single, integrated pharmacodynamic model to the data is limited. The most tractable solution in this case was to split the data into two groups and model them separately: the first model quantifying the rate of increase in nAbs and the second model describing the subsequent decay. The antibody decay was based on ref. ${ }^{37}$. While this two-stage approach did not allow data from the 'stimulation' phase to inform the model fit of the 'decay' phase-and vice versa-it did enable accurate and quantitative characterization of both the stimulation and decay dynamics, which have not been characterized for EBOV disease before this study, and which may be used to inform future work in this area and other impactful viral diseases such as COVID-19.

\section{Statistical analysis}

Statistical analyses of data were implemented using GraphPad Prism 6.0 software. Unpaired sample comparisons were conducted for all data; individual figure legends state the corresponding statistical tests performed. These include parametric and non-parametric $t$-tests (Student's $t$-test and Mann-Whitney $U$-test); parametric and non-parametric ANOVAs (ordinary ANOVA and Kruskal-Wallis test). ${ }^{*} P<0.05,{ }^{* *} P<0.01$, ${ }^{* * *} P<0.001,{ }^{* * * *} P<0.0001$.

\section{Reporting summary}

Further information on research design is available in the Nature Research Reporting Summary linked to this paper.

\section{Data availability}

All datasets generated during and/or analysed during the current study are available from the corresponding author on reasonable request. Source data are provided with this paper.

44. Scott, J. T. \& Semple, M. G. Ebola virus disease sequelae: a challenge that is not going away. Lancet Infect. Dis. 17, 470-471 (2017).

45. Platt, E. J., Bilska, M., Kozak, S. L., Kabat, D. \& Montefiori, D. C. Evidence that ecotropic murine leukemia virus contamination in TZM-bl cells does not affect the outcome of neutralizing antibody assays with human immunodeficiency virus type 1. J. Virol. 83, 8289-8292 (2009).

46. Takeuchi, Y., McClure, M. O. \& Pizzato, M. Identification of gammaretroviruses constitutively released from cell lines used for human immunodeficiency virus research. J. Virol. 82, 12585-12588 (2008).

47. Wei, X. et al. Emergence of resistant human immunodeficiency virus type 1 in patients receiving fusion inhibitor (T-20) monotherapy. Antimicrob. Agents Chemother. 46 1896-1905 (2002).
48. Derdeyn, C. A. et al. Sensitivity of human immunodeficiency virus type 1 to the fusion inhibitor T-2O is modulated by coreceptor specificity defined by the V3 loop of gp120. J. Virol. 74, 8358-8367 (2000).

49. Platt, E. J., Wehrly, K., Kuhmann, S. E., Chesebro, B. \& Kabat, D. Effects of CCR5 and CD4 cell surface concentrations on infections by macrophagetropic isolates of human immunodeficiency virus type 1. J. Virol. 72, 2855-2864 (1998).

50. Biek, R., Walsh, P. D., Leroy, E. M. \& Real, L. A. Recent common ancestry of Ebola Zaire virus found in a bat reservoir. PLoS Pathog. 2, e90 (2006).

51. Connor, R. I., Chen, B. K., Choe, S. \& Landau, N. R. Vpr is required for efficient replication of human immunodeficiency virus type-1 in mononuclear phagocytes. Virology 206, 935-944 (1995).

52. Wei, X. et al. Antibody neutralization and escape by HIV-1. Nature 422, 307-312 (2003).

53. Neely, M. N., van Guilder, M. G., Yamada, W. M., Schumitzky, A. \& Jelliffe, R. W. Accurate detection of outliers and subpopulations with Pmetrics, a nonparametric and parametric pharmacometric modeling and simulation package for R. Ther. Drug Monit. 34, 467-476 (2012).

54. R Core Team. R: A Language and Environment for Statistical Computing v. 3.1.0 (R Foundation for Statistical Computing, 2014).

55. De Cock, R. F. W. et al. Role of modelling and simulation in paediatric clinical research. Eur. J. Clin. Pharmacol. 67, S5-S16 (2011).

56. Aarons, L. et al. Role of modelling and simulation in phase I drug development. Eur. J. Pharm. Sci. 13, 115-122 (2001).

57. Roberts, J. A. et al. Plasma and target-site subcutaneous tissue population pharmacokinetics and dosing simulations of cefazolin in post-trauma critically ill patients. J. Antimicrob. Chemother. 70, 1495-1502 (2015).

58. Grau, S. et al. Plasma and peritoneal fluid population pharmacokinetics of micafungin in post-surgical patients with severe peritonitis. J. Antimicrob. Chemother. 70, 2854-2861 (2015).

59. Mould, D. R. \& Upton, R. N. Basic concepts in population modeling, simulation, and model-based drug development-part 2: introduction to pharmacokinetic modeling methods. CPT Pharmacometrics Syst. Pharmacol. 2, e38 (2013).

Acknowledgements We thank colleagues variously for their support and encouragement: the Sierra Leone Association of Ebola Survivors (Freetown, Sierra Leone); the members of the Convalescent Products and Allied Therapy Intervention Technical Committee; the Research Ethics Committee and the Pharmacy Board Committee (all Ministry of Health and Sanitation, Republic of Sierra Leone); staff in Virus Reference Department Public Health England for handling and clearing samples from quarantine; G. McCann and L. Matthews for project management; I. Bates for expertise in strengthening transfusion services; W. A. Brooks; M. P. Kieny; C. Burm and D. Arango; N. F. Walker; A. Jones; colleagues from the World Health Organization (WHO); and the International Severe Acute Respiratory and Emerging Infection Consortium (ISARIC). Proofreading support provided by M. de Baar and graphical support provided by S. Yee. The study "Convalescent plasma for early Ebola virus disease in Sierra Leone (Ebola CP)" (ISRCTN13990511 and PACTR201602001355272) was supported by the Wellcome Trust (Award 106491) and Bill and Melinda Gates Foundation; Public Health England Ebola Emergency Response; and the Blood Safety Programme, National Health Service Blood and Transplant. J.T.S. was supported by the Wellcome Trust. M.G.S. and J.T.S. were supported by the UK National Institute for Health Research Health Protection Research Unit in Emerging and Zoonotic Infections at the University of Liverpool. The funders had no role in the collection and analysis of the samples, in the interpretation of data, in writing the report, or in the decision to submit the paper for publication.

Author contributions G.P., W.A.P., J.T.S. and M.G.S. initiated and designed the study. C.A., R.S.T., J.T.S., R.S., R.J.D. and R.G. collected data and/or performed the analysis. C.A., G.P. and W.A.P. wrote the manuscript. M.G.S., J.T.S., G.P., R.S.T., R.J.D. and W.A.P. edited the manuscript. M.G.S. sourced the funding and is Ebola-CP Consortium Lead Investigator. All authors were critical for study delivery whether through recruitment, coordination, collection of participant data and material, assay development or analysis of samples. All authors read and approved the contents of the manuscript. GSK was not involved in the design, conduct or analysis of the study.

Competing interests The authors declare no competing interests.

\section{Additional information}

Supplementary information The online version contains supplementary material available at https://doi.org/10.1038/s41586-020-03146-y.

Correspondence and requests for materials should be addressed to G.P.

Peer review information Nature thanks Jayajit Das and the other, anonymous, reviewer(s) for their contribution to the peer review of this work.

Reprints and permissions information is available at http://www.nature.com/reprints. 
a

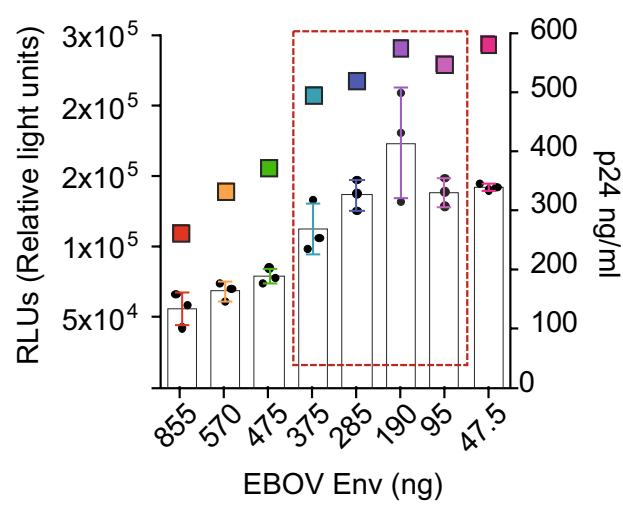

C

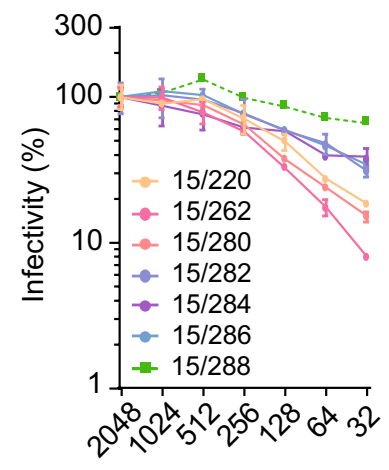

Plasma dilutions

e

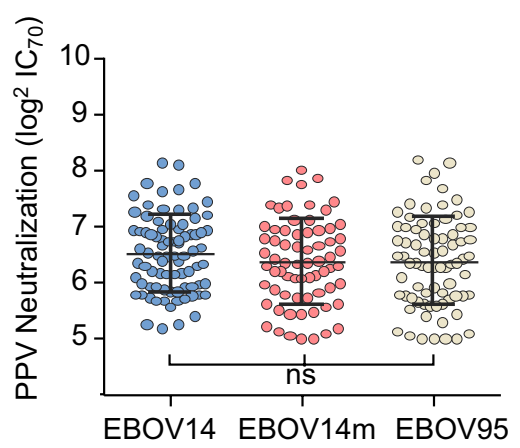

9

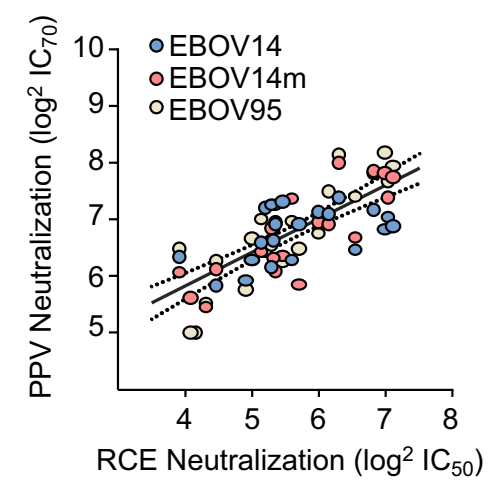

b

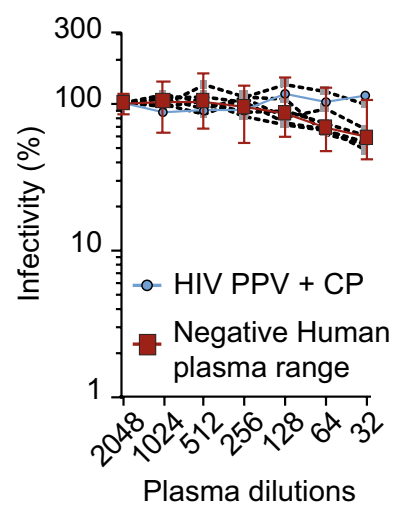

Plasma dilutions

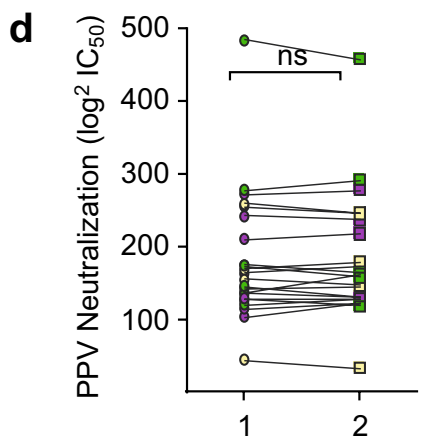

Experiment number

f

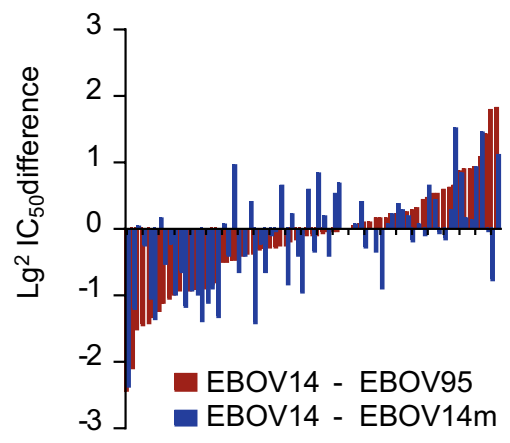

h

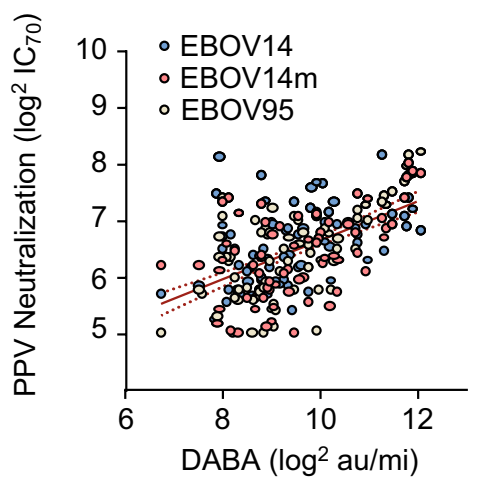

Extended Data Fig. 1 |See next page for caption. 
Extended Data Fig. 1 | Development and characterization of Ebola pseudoparticle virus. a, Variant pEBOV14-GP plasmid concentrations were transfected alongside $2000 \mathrm{ng}$ of pSG3-HIV-1 backbone. The resulting pseudotyped virus, quantified by a HIV-1-p24 capsid ELISA (squares), was tested for infectivity in TZM-bl cells as measured by luciferase activity (data are mean \pm s.d.). The red marked square identifies the glycoprotein concentrations that can be used in the assay. $\mathbf{b}$, Inhibition profiles with negative plasma donated from six individuals (grey squares), indicating no specific plasma inhibition during the neutralization assay. All negative assays and plasmas were combined to define the range within which negative plasma control were acceptable (red squares) thus defining a valid assay. The blue line shows the lack of reactivity on the HIV-1-enveloped pseudo-typed virus by EBOV neutralizing convalescent plasma (CP) (squares and circles indicate the median and the vertical lines the standard error).c, Neutralization profiles of pEBOV14-GP by the WHO reference panel of anti-EBOV CP. The standard identifiers are shown.

d, Reproducibility of the neutralization assay determined by measuring the $\mathrm{IC}_{50}$ of CP on the three EBOV isolates (yellow-pEBOV14-GP, purple-pEBOV95-GP and green-pEBOV14m-GP). The two-tailed parametric paired $t$-test was used. e, Neutralization potential of CPs against three virus strains ( $\mathrm{pEBOV14-}$ $\mathrm{GP} / n=83, \mathrm{pEBOV} 95-\mathrm{GP} / n=69$ and $\mathrm{pEBOV} 14 \mathrm{~m}-\mathrm{GP} / n=77$ ) expressed in $\mathrm{IC}_{70}$ (data are presented as mean values \pm s.d. Kruskal-Wallis test was performed).f, delta$\mathrm{IC}_{70}$ neutralization titres between virus strain pairs by each post-cure study participant. g, Positive association between PPV IC ${ }_{70}$ titres the live virus plaque reduction neutralization test (PRNT). h, Positive association between $\mathrm{PPVIC}_{70}$ neutralization titres and the double antigen bridging assay (DABA). 
a

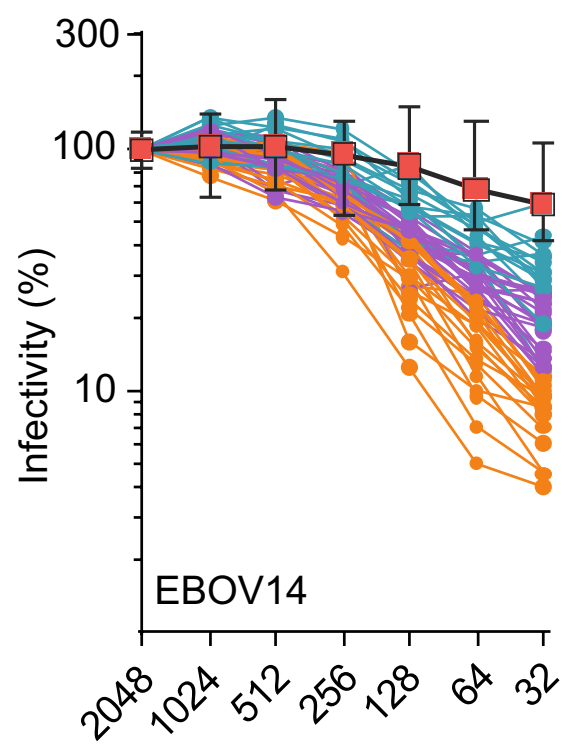

b

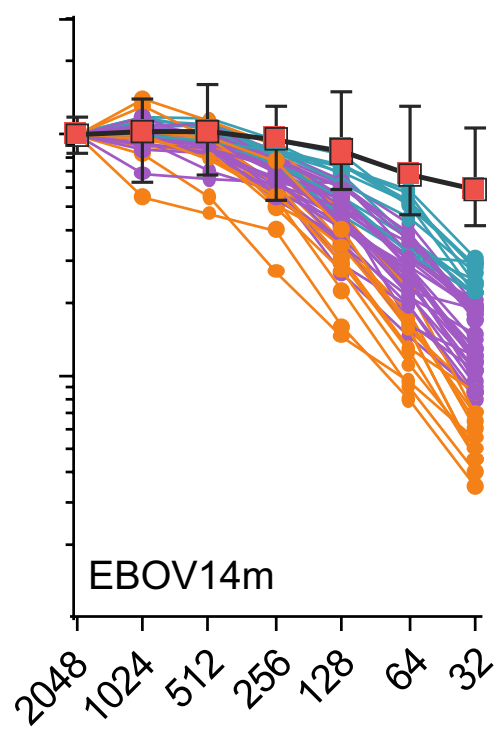

Plasma dilutions
C

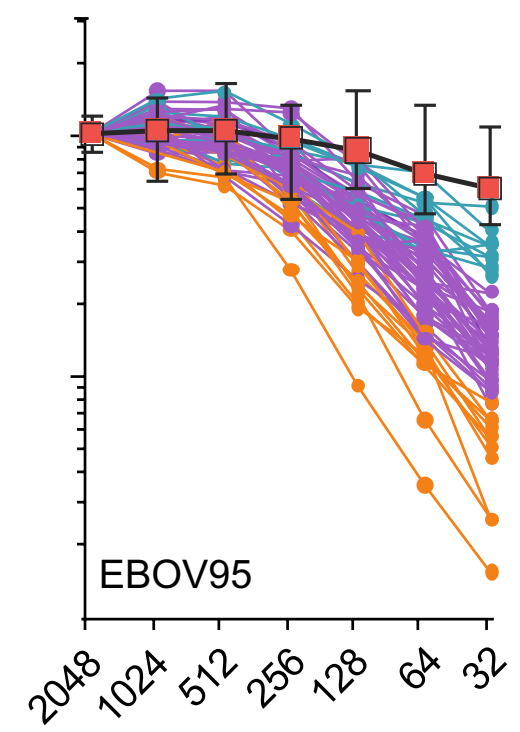

d

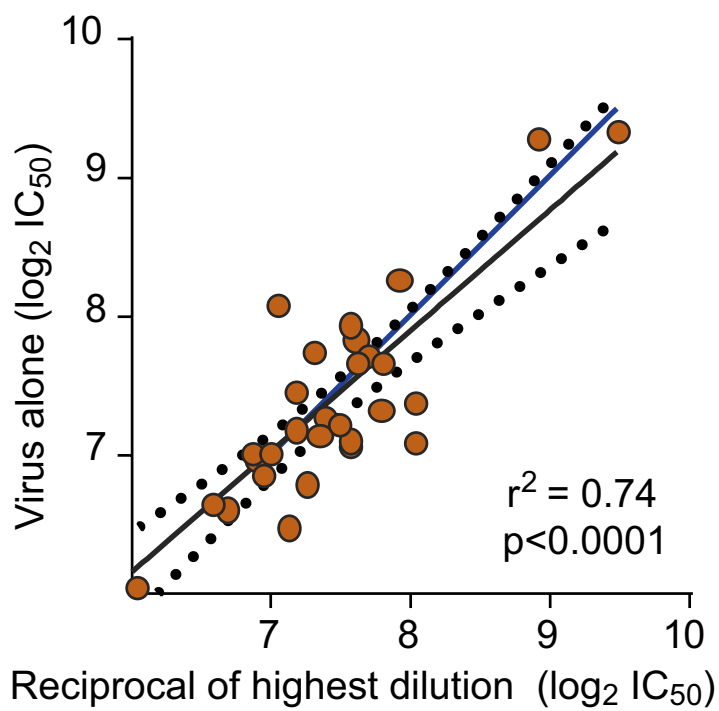

Extended Data Fig. 2 | Pseudo-particle virus neutralization profiles by convalescent plasma. a-c, Anti-EBOV14-GP (a; $n=92)$, anti-EBOV14m-GP $(\mathbf{b} ; n=70)$ and anti-EBOV95-GP (c; $n=76)$ neutralization curves using serial dilutions of CP inhibiting PPV cell entry, as described in methods. The plasma samples were deciphered as possessing low (blue), intermediate (magenta) or high (orange) neutralization to demonstrate the similar profiles of the three virus glycoproteins studied. The red square curve indicates the range of inhibition by control plasma. d, Comparison of the analyses $(n=30)$ (i) considering the $0 \%$ inhibition value whenever two reciprocal consecutive high plasma dilutions produced equal infection levels, and (ii) considering $0 \%$ inhibition as the infection values of virus in the absence of convalescent plasma performed in each individual experiment. The Pearson correlation coefficients were computed. 

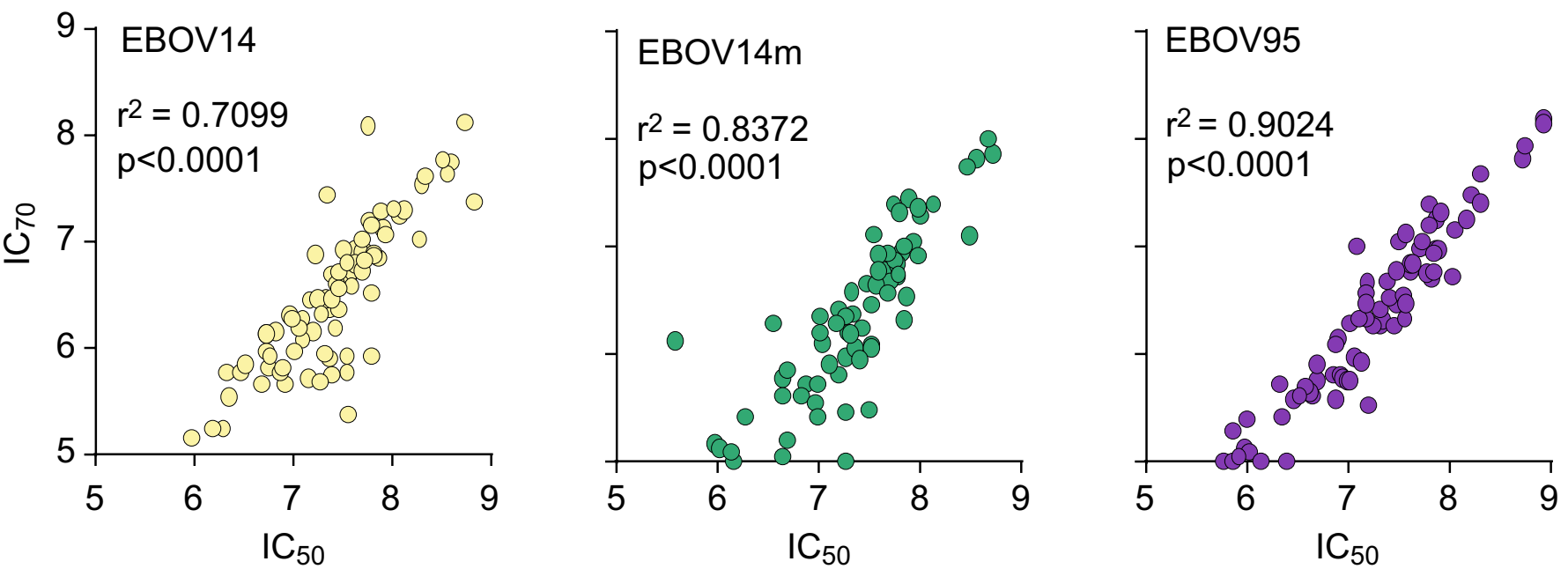

Extended Data Fig. 3 | Association of $\mathrm{IC}_{50}$ and $\mathrm{IC}_{70}$ neutralizing dilutions of the post-cure plasma samples inhibiting cell entry of pseudotyped virus particles harbouring the variant EBOV GP molecules. The Pearson correlation coefficients were computed. 
18

$\square \mathrm{NP}$

$\rightarrow$ EBOV14

$\rightarrow$ EBOV95

- VP40

$\square \mathrm{GP}$
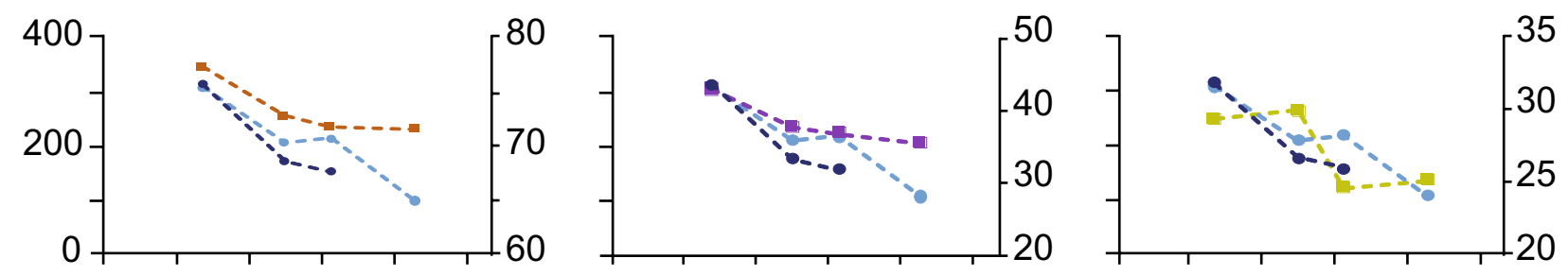

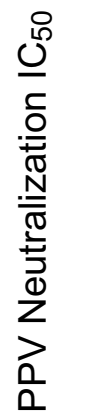

19
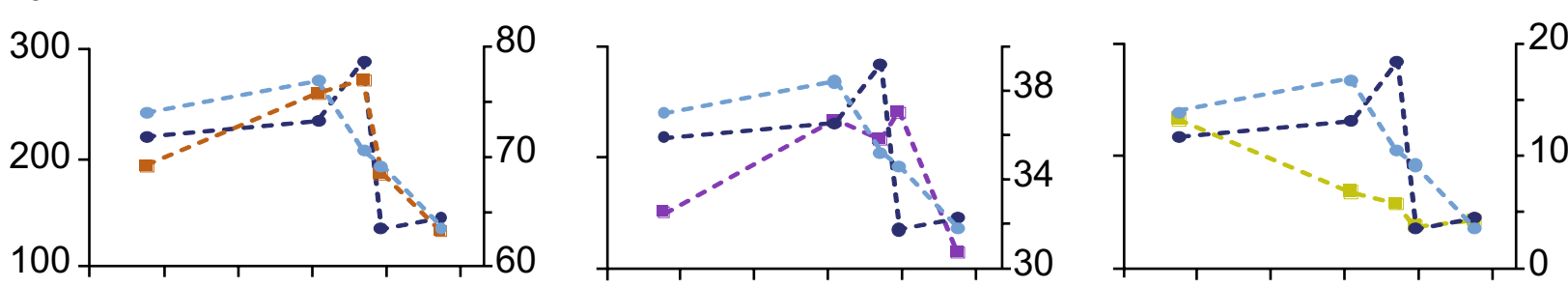

$\frac{w}{8}$
$\frac{0}{\hat{1}}$
$\frac{0}{0}$
$\frac{m}{2}$
$\frac{m}{2}$

\section{1}
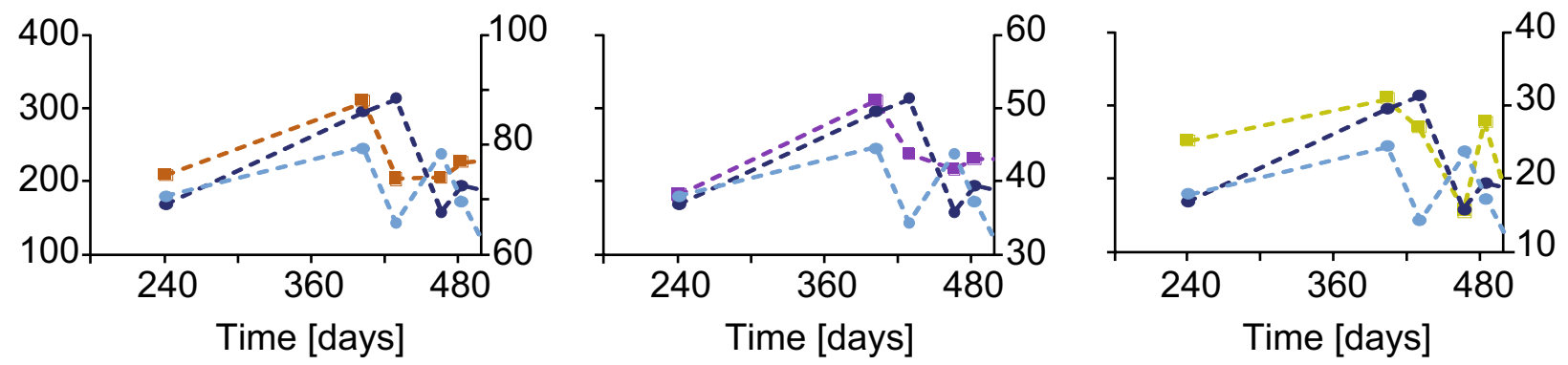

Extended Data Fig. 4 | Longitudinal post convalescence $n A b$ variation in the plasma of individuals 18, 19 and 21 demonstrated by pseudotyped virus particle neutralization. Anti-EBOV14-GP (light blue) and anti-EBOV95-GP

detection of antibody against the nucleoprotein [NP] (brown squares), the viral matrix protein 40 [VP40] (purple squares) and the glycoprotein [GP] (green squares). 
18

IgG Capture EIA EBOV14

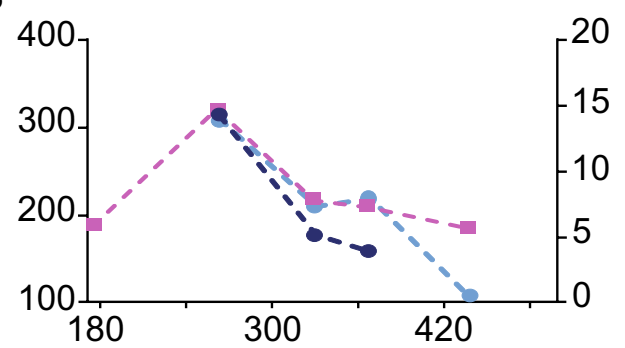

19

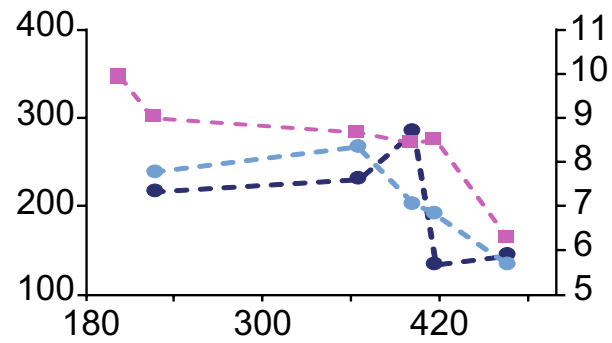

21

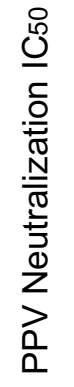

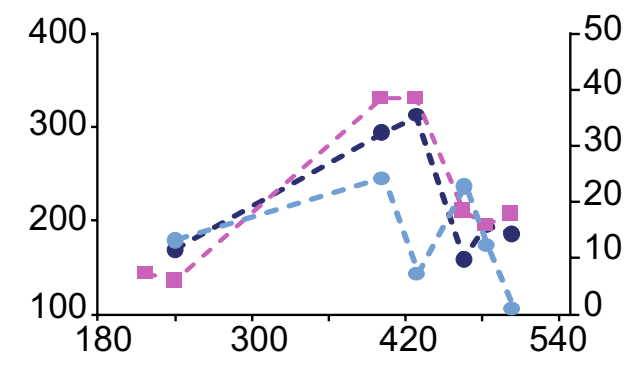

45

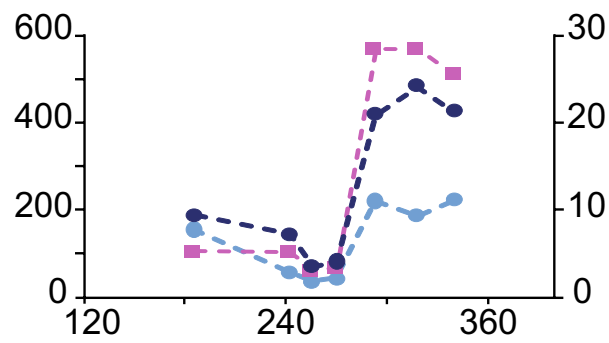

49

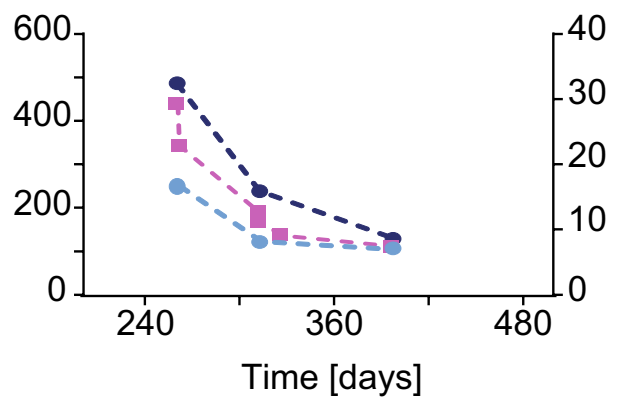

Extended Data Fig. 5 | Longitudinal G-capture and competitive EIAs performed using plasma from individuals $18,19,21,45$ and 49 against the glycoprotein as previously described. The longitudinal G-capture (pink) and competitive (green) ElAs were performed against the glycoprotein as
EBOV95 Competitive EIA
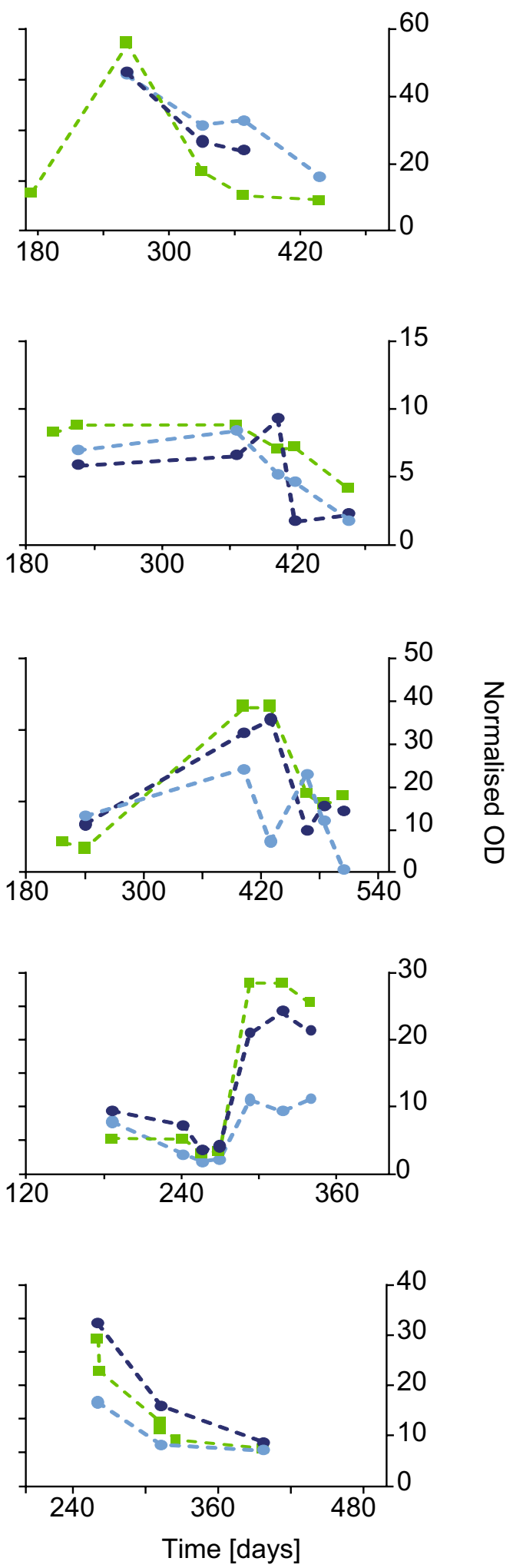

previously described ${ }^{21}$. The antibody reactivities were overlaid with pseudotyped virus particle $\mathrm{IC}_{50}$ neutralization values against EBOV14-GP (light blue) and EBOV95-GP (dark blue). 


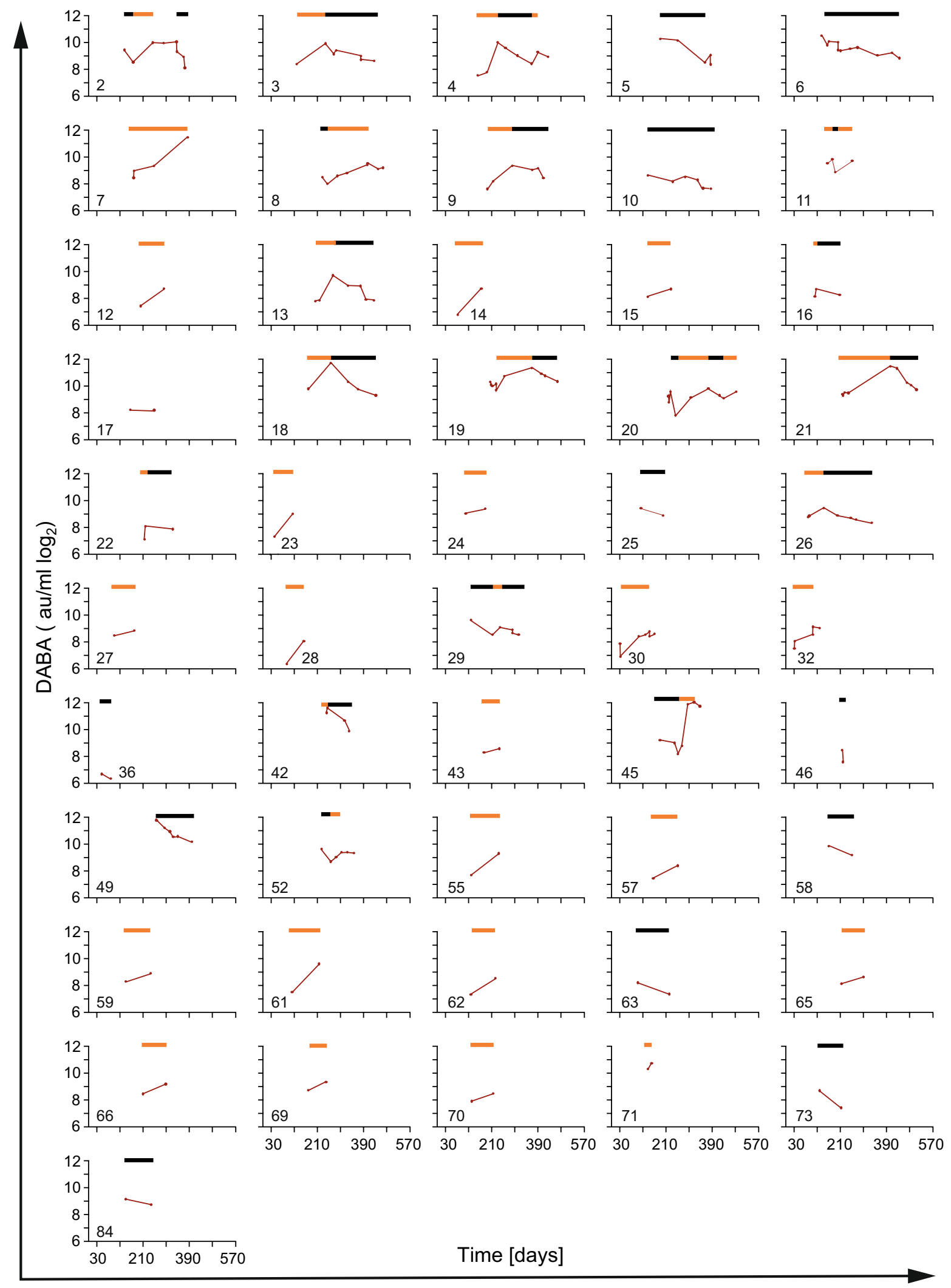

Extended Data Fig. 6 | Total antibody reactivity as measured by double antigen bridging assay (DABA) (average of a duplicate measurement) for the Ebola post-cure cohort participants with longitudinal follow up
( $\geq 2$ data points, $n=51$ ) demonstrating decline-restimulation-decline (in any order) of antibody reactivity over time. Decline is indicated by a black line and restimulation by a yellow horizontal line. 


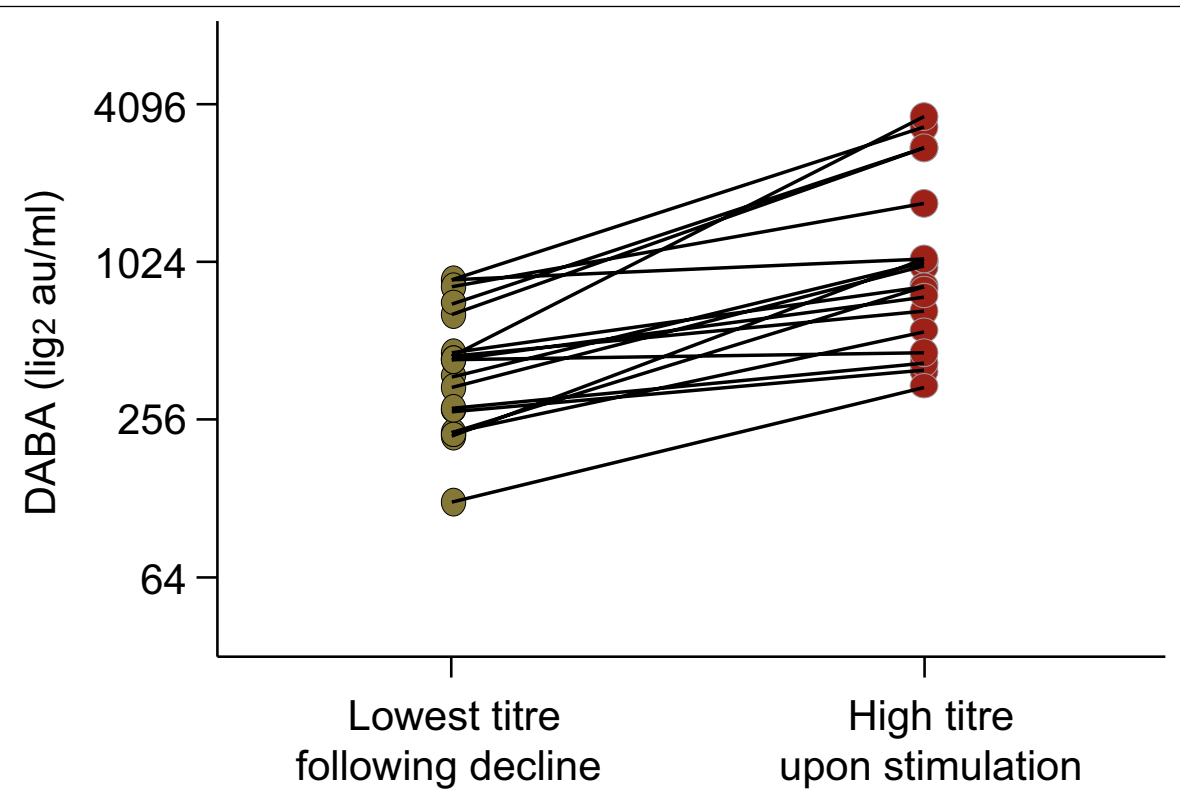

Extended Data Fig. 7 | Total antibody reactivity as measured by double antigen bridging assay (DABA). 'Lowest titre following decline' is the last

stimulation' is the subsequent point demonstrating antibody stimulation. Two-tailed parametric paired $t$-test $(P=0.0014)$. 


\section{Article}
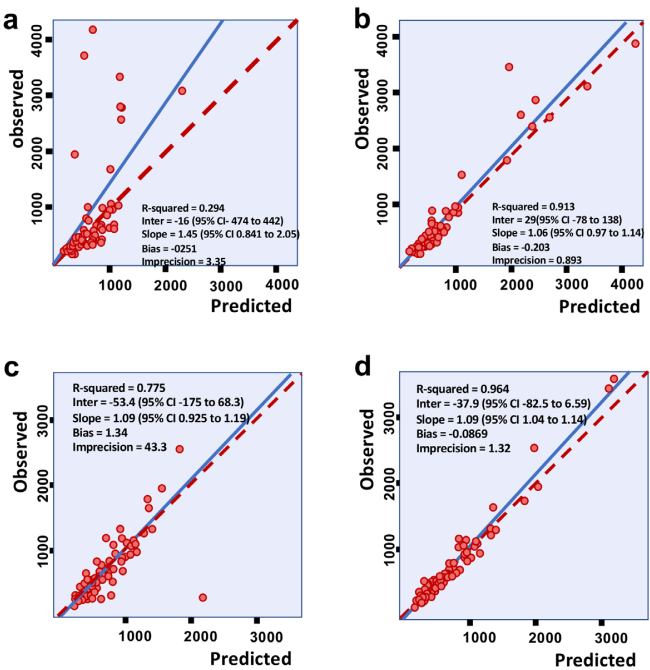

Extended Data Fig. $8 \mid$ Observed versus predicted plots for the growth and decay models as determined by the DABA assay. a, b, Plots for selected logistic growth model for antibody stimulation. a, Population predicted values. b, Individual predicted values. c, d, Plots for selected two-compartment decay model with saturable recycling for antibody stimulation. c, Population predicted values. d, Individual predicted values. Solid red circles represent the individual observed or model-predicted Ab values. Solid blue line and dotted red line represent the line of regression and line of unity, respectively. 


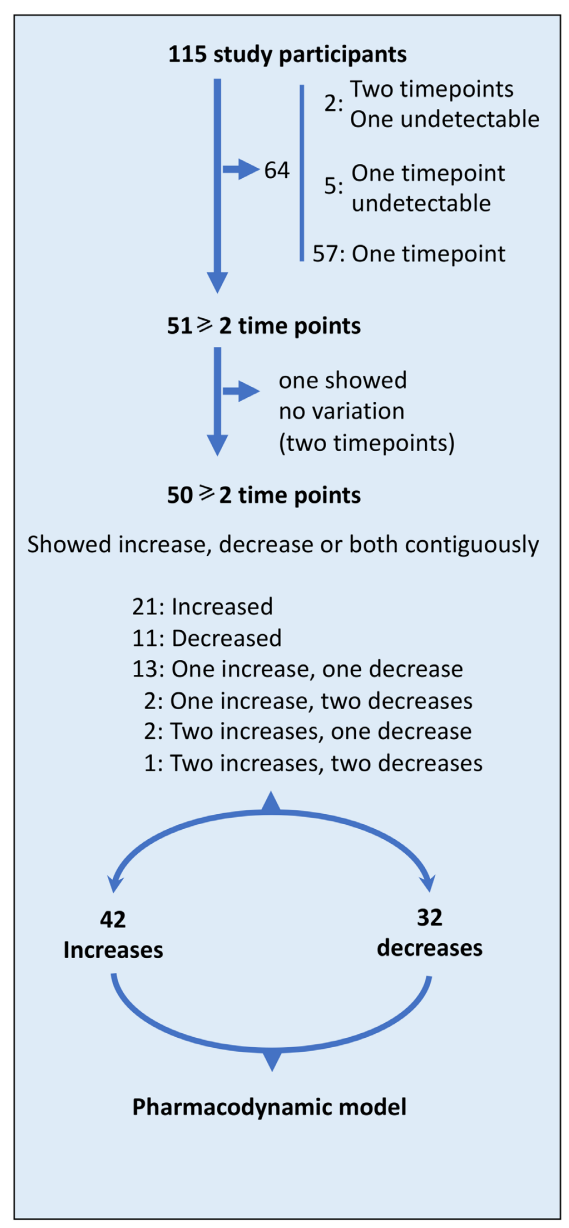

Extended Data Fig. 9|Flow diagram describing the observed antibody decrease and increase events as measured by DABA. These were used to develop the compartmental population pharmacodynamic models. 


\section{nature research}

Corresponding author(s): Georgios Pollakis

Last updated by author(s): Jan 10, 2021

\section{Reporting Summary}

Nature Research wishes to improve the reproducibility of the work that we publish. This form provides structure for consistency and transparency in reporting. For further information on Nature Research policies, see Authors \& Referees and the Editorial Policy Checklist.

\section{Statistics}

For all statistical analyses, confirm that the following items are present in the figure legend, table legend, main text, or Methods section. n/a Confirmed

$\bigotimes$ The exact sample size $(n)$ for each experimental group/condition, given as a discrete number and unit of measurement

\ A statement on whether measurements were taken from distinct samples or whether the same sample was measured repeatedly

$\triangle$ The statistical test(s) used AND whether they are one- or two-sided

Only common tests should be described solely by name; describe more complex techniques in the Methods section.

Х $\square$ A description of all covariates tested

Х $\square$ A description of any assumptions or corrections, such as tests of normality and adjustment for multiple comparisons

$\triangle$ A full description of the statistical parameters including central tendency (e.g. means) or other basic estimates (e.g. regression coefficient)

AND variation (e.g. standard deviation) or associated estimates of uncertainty (e.g. confidence intervals)

For null hypothesis testing, the test statistic (e.g. $F, t, r)$ with confidence intervals, effect sizes, degrees of freedom and $P$ value noted

Give $P$ values as exact values whenever suitable.

$\bigotimes$ For Bayesian analysis, information on the choice of priors and Markov chain Monte Carlo settings

Х $\square$ For hierarchical and complex designs, identification of the appropriate level for tests and full reporting of outcomes

Х $\square$ Estimates of effect sizes (e.g. Cohen's $d$, Pearson's $r$ ), indicating how they were calculated

Our web collection on statistics for biologists contains articles on many of the points above.

\section{Software and code}

\section{Policy information about availability of computer code}

\section{Data collection No software was used}

Data analysis PRISM version 6.07, Pmetrics version 1.4.1 and R3.2.2

For manuscripts utilizing custom algorithms or software that are central to the research but not yet described in published literature, software must be made available to editors/reviewers. We strongly encourage code deposition in a community repository (e.g. GitHub). See the Nature Research guidelines for submitting code \& software for further information.

\section{Data}

Policy information about availability of data

All manuscripts must include a data availability statement. This statement should provide the following information, where applicable:

- Accession codes, unique identifiers, or web links for publicly available datasets

- A list of figures that have associated raw data

- A description of any restrictions on data availability

All datasets generated and/or analysed during the study are available from the corresponding authors on reasonable request 2014 epidemic strain ( $p$ EBOV14-GP) (GenBank accession number: KP096421)

1995 Kikwit strain (pEBOV95-GP) (GenBank accession number: KC242799)

Plaque reduction strain EBOV Makona (GenBank accession number: KJ660347)

DABA: Zaire Ebolavirus isolate Yambuku-Mayinga (GenBank accession number: NC_002549.1)

Ebolavirus glycoprotein: https://www.ncbi.nlm.nih.gov/nuccore/?term=Ebolavirus 
Please select the one below that is the best fit for your research. If you are not sure, read the appropriate sections before making your selection.

$\bigotimes$ Life sciences $\quad \square$ Behavioural \& social sciences $\square$ Ecological, evolutionary \& environmental sciences

For a reference copy of the document with all sections, see nature.com/documents/nr-reporting-summary-flat.pdf

\section{Life sciences study design}

All studies must disclose on these points even when the disclosure is negative.

Sample size All participants ( $n=115)$ were tested using DABA, blocking EIA and IgG capture immunoassays. PPV antibody neutralisation assays were performed with a subset of participants not selected on any criteria $(n=52)$ and the compartmental population pharmacodynamics model was developed on the more replete DABA dataset $(n=115)$ using those participants with longitudinal data $(n=51)$.

This analysis was "post-hoc" so the study was not powered to meet any required precision in the PD endpoints. For sparse PK or PD sampling, individual derived parameters may not be possible, hence a population modeling approach is required as used in analysis, and standard statistical sample size calculations may not be valid. Furthermore, the credibility interval ( $95 \% \mathrm{Cl}$ ) of parameters estimates don't overlap zero, indicating an acceptable level of precision.

Data exclusions None

Replication When the assay was shown to be reliable and robust not all findings were replicated. All assays were performed in duplicate or triplicate and were replicated when the values were discrepant.

Randomization Random allocation is not relevant to this study because there is no allocation of intervention. This is an observational study.

Blinding $\quad$ Testing of patient convalescent plasma in wild type replication virus was performed blindly and unblinded for comparison with pseudo-typed virus particle neutralisation upon experimental completion.

\section{Reporting for specific materials, systems and methods}

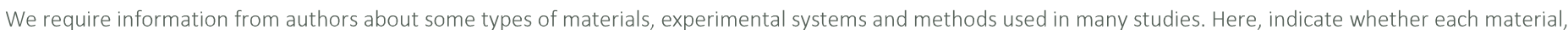

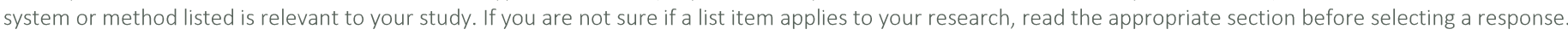

\begin{tabular}{|c|c|c|c|}
\hline \multicolumn{2}{|c|}{ Materials \& experimental systems } & \multicolumn{2}{|c|}{ Methods } \\
\hline $\mathrm{n} / \mathrm{a}$ & Involved in the study & $\mathrm{n} / \mathrm{a}$ & Involved in the study \\
\hline & $\bigotimes$ Antibodies & Х & ChIP-seq \\
\hline & Xukaryotic cell lines & Х & Flow cytometry \\
\hline Х & Palaeontology & Х & MRI-based neuroimaging \\
\hline Х & Animals and other organisms & & \\
\hline & Х Human research participants & & \\
\hline & Х Clinical data & & \\
\hline
\end{tabular}

\section{Antibodies}

Antibodies used

p24 coating antibody (polyclonal sheep anti-HIV-1-p24 gag) (Aalto Bio Reagents Ltd: D7320) alkaline phosphatase conjugate of mouse monoclonal anti-HIV-1-p24 (Boehringer Mannheim: 1089-161) Anti- EBOV Convalescent Plasma Pool - Sierra Leone (International Reference Reagent: Code 15/262) WHO Anti-EBOV Convalescent Plasma (International Reference Panel: Code 16/344)

Anti-EBOV plasma, human (WHO Reference Reagent: Code 15/220)

Murine monoclonal antibody 4G7 (gifted by Gary Kobinger, Winnipeg, Canada)

Polyclonal rabbit anti-sera to human $\gamma$-Fc (Jackson Immuno Research Cat: 309-005-008)

Anti-EBOV GP monoclonal antibody (IBT Bioservices c13C6FR1: Code 0201-023)

Rabbit anti-EBOV GP pAb (IBT Bioservices: Code 0301-015)

Rabbit anti-EBOV NP pAb (IBT Bioservices: Code 0301-012)

Rabbit anti-EBOV VP40 pAb (IBT Bioservices: Code 0301-010)

Validation

These antibodies have been validated by the manufacturer. Please see the manufacturer or the provider home pages for the specific citations for each antibody. 
Policy information about cell lines

Cell line source(s)

HEK293T (ATCC ${ }^{\oplus}$ CRL-3216 ${ }^{\text {TM }}$ )

TZM-bl2-6 cells (HIV reagent Programme)

Vero E6 cells (ECACC: 85020206)

Authentication

None were authenticated

Mycoplasma contamination

Commonly misidentified lines

(See ICLAC register)

All cell lines used were deemed Mycoplasma free

No commonly misidentified cells lines were use

\section{Human research participants}

Policy information about studies involving human research participants

Population characteristics

We examined the cohort of donors for the Ebola CP trial in Sierra Leone.

The trial adhered to interim WHO guidance that CP donors be selected from PCR-confirmed Ebola cases who were clinically asymptomatic 28 days after discharge from an Ebola Treatment Unit (ETU), where discharge was based on clinical grounds and supported by two negative PCR results taken at least 48 hours apart. A minimum interval for repeated plasma donation was set at two weeks for donor wellbeing. No fixed schedule was set for returning to donate.

Every donation was preceded by a donor health check and testing for Hepatitis B \& C, HIV and Syphilis.

Apheresis used the Haemonetics Plasma Collection System PCS2 ${ }^{\circledR}$ (Haemonetics Corp., Braintree MA USA), where the anticoagulant was Citrate Phosphate Double Dextrose Solution. Pathogen reduction used the INTERCEPT'M processing system (Cerus Corp., Concord CA USA), where freshly collected plasma was mixed with amotosalen $\mathrm{HCl}$ and then exposed to ultraviolet A (UVA) transillumination.

Recruitment

Recruitment was supported by the Sierra Leone Association of Ebola Survivors. People with Ebola survivor certificates and survivors discharged from the ETU at 34 Military Hospital (MH34), Freetown were invited to attend a recruitment clinic at MH34. Those who gave consent underwent an interview and examination. Those who were unwell or with possible Post Ebola Syndrome were referred to the Ebola Survivors' Clinic at MH34. Healthy candidate donors were referred to the Safe Blood Service blood bank at Connaught Hospital, Freetown for formal donor assessment in line with national guidelines. Plasma was donated April 2015-February 2016 in the blood bank donation room and immediately processed in the blood bank laboratory. For humanitarian reasons the priority was to establish rapidly a bank of CP with high Ebola antibody reactivity. Measurement in the blood bank was possible using G-capture and Competitive EIAs. Repeated donations were invited from those donors whose antibody reactivity was in the upper quartile when reviewed in temporal groups of approximately 20 donors, though accepted from any donor who wished to donate repeatedly.

At each donation, plasma aliquots $(5 \mathrm{~mL})$ were stored in Sierra Leone at $-20^{\circ} \mathrm{C}$ and later, with specific permission, flown to England for further determination of Ebola antibody reactivity. All samples were then confirmed to be free of detectable EBOV RNA before further analysis in a BSL2 laboratory.

These subjects fitted recruitment criteria for convalescent plasma-donors and as such were checked for their fitness to donate. This may have resulted in a fitter population than the general Ebola survivor population. Consequently, the findings of this study may be an underestimation of what would be observed in the general population. Age or gender are not included in the analysis since the majority of participants were between $22 y$ and $35 y$ and there was no age difference between male and female.

No selection was considered for healthy volunteers aside the fact that they were not exposed to the Ebola virus and were all, otherwise, healthy lab workers.

Ethics oversight

The Ebola CP trial was approved by the Sierra Leone Ethics and Scientific Review Committee and authorized by Pharmacy Board of Sierra Leone (PBSL/CTAN/MOHS-CSTO01). The protocol was also approved by the ethics committee of the London School of Hygiene and Tropical Medicine, and the University of Liverpool as Sponsor.

The protocol for healthy volunteer controls was approved by the University of Liverpool as sponsor (RETH000685)

All participants have signed an informed consent form available upon request.

Note that full information on the approval of the study protocol must also be provided in the manuscript.

\section{Clinical data}

Policy information about clinical studies

All manuscripts should comply with the ICMJE guidelines for publication of clinical research and a completed CONSORT checklist must be included with all submissions.

Clinical trial registration

Study protocol

Data collection
Convalescent plasma for early Ebola virus disease in Sierra Leone (Ebola CP) - ISRCTN13990511 \& PACTR201602001355272

On request to the CI Prof. M. G. Semple m.g.semple@liverpool.ac.uk

This work does not relate to the outcome of the trial subjects i.e. those been treated for Ebola Virus Disease as part of the trial, but to the characteristics of the cohort of volunteers who donated plasma by apheresis for that trial. Donor data was collected as desribed in this paper and a previous publication cited in the body of the work submitted (Tedder RS et al. Transfusion 2018) 
